\title{
Evolution of south seasonal cap during Martian spring: Insights from high-resolution observations by HiRISE and CRISM on Mars Reconnaissance Orbiter
}

\author{
A. Pommerol, ${ }^{1}$ G. Portyankina, ${ }^{1}$ N. Thomas, ${ }^{1}$ K.-M. Aye, ${ }^{1}$ C. J. Hansen, ${ }^{2}$ \\ M. Vincendon, ${ }^{3,4}$ and Y. Langevin ${ }^{4}$
}

Received 14 December 2010; revised 16 May 2011; accepted 27 May 2011; published 25 August 2011.

[1] We use data from the High Resolution Imaging Science Experiment (HiRISE) camera and the Compact Reconnaissance Imaging Spectrometer for Mars (CRISM) imaging spectrometer onboard the Mars Reconnaissance Orbiter to follow the evolution of the appearance and composition of 12 regions of the south polar layered deposits from spring to summer time. We distinguish three steps in the evolution of the volatile layer: a decrease of both $\mathrm{CO}_{2}$ band strength and albedo until $\mathrm{Ls}=190^{\circ}-210^{\circ}$, a significant increase in both until Ls $=240^{\circ}-260^{\circ}$ and finally a rapid decrease until the complete defrosting of the ground. In contrast, the water ice band displays a more monotonic decrease. Analysis of HiRISE color images acquired simultaneously with CRISM data allows a plausible interpretation of this evolution. In early springtime $\left(\mathrm{Ls}<200^{\circ}\right)$, intense jet activity results in deposition of fans of large mineral grains and a wide spatial distribution of fine grains. The small-scale topography controls the presence and location of the jets by allowing more solar energy to be collected on slopes. Grains from the dust fans warm and sink through the $\mathrm{CO}_{2}$ layer, resulting in a bluish color at the locations of the fans around $\mathrm{Ls}=190^{\circ}-210^{\circ}$. As the atmosphere warms up, the surface of the ice layer sublimes and releases dust and water, resulting in its brightening. The last phase of the process consists in a progressive defrosting resulting in a patchwork of frozen and unfrozen areas.

\begin{abstract}
Citation: Pommerol, A., G. Portyankina, N. Thomas, K.-M. Aye, C. J. Hansen, M. Vincendon, and Y. Langevin (2011), Evolution of south seasonal cap during Martian spring: Insights from high-resolution observations by HiRISE and CRISM on Mars Reconnaissance Orbiter, J. Geophys. Res., 116, E08007, doi:10.1029/2010JE003790.
\end{abstract}

\section{Introduction}

[2] The south polar regions of Mars are among the most extensively studied areas of the planet. There is a strong interest in these regions because of the prominent role that they play in the global volatile cycles [James et al., 1992] and the wide range of dynamic processes that occur on the surface [Malin et al., 1998]. Furthermore, repeated observations with high temporal resolution are readily available because of the quasi-polar orbits of recent Martian orbiters. The use of a number of complementary remote sensing methods has already permitted in-depth analyses of the nature and seasonal evolution of the south polar terrains.

[3] A large part of the south polar terrains belongs to the South Polar Layered Deposits (SPLD) Amazonian unit that

\footnotetext{
${ }^{1}$ Physikalisches Institut, Universität Bern, Bern, Switzerland.

${ }^{2}$ Planetary Science Institute, Tucson, Arizona, USA.

${ }^{3}$ Department of Geological Science, Brown University, Providence, Rhode Island, USA.

${ }^{4}$ Institut d'Astrophysique Spatiale, CNRS/Université Paris XI, Orsay, France.

Copyright 2011 by the American Geophysical Union. 0148-0227/11/2010JE003790
}

lies on top of the Archean southern plateau [Tanaka and Scott, 1987; Tanaka and Kolb, 2001a, 2001b]. The SPLD consist of superposed thin layers of water ice contaminated by a variable amount of mineral dust $(0-15 \mathrm{wt} \%$ [Plaut et al., 2007; Zuber et al., 2007]). The total thickness of this unit reaches $3.7 \mathrm{~km}$ close to the South Pole. Its surface age is estimated to be around $10 \mathrm{Myr}$, and its formation is probably related to the past evolution of the planet's obliquity [Herkenhoff and Plaut, 2000]. The ice-rich terrains are overlain by a desiccated layer that is optically thick, as indicated by the absence of water ice features in near-infrared spectra, except in limited areas within and around the bright permanent polar cap [Bibring et al., 2004; Douté et al., 2007]. Detection of abundant hydrogen in the uppermost meter of the SPLD $\left(>50 \mathrm{wt} \% \mathrm{H}_{2} \mathrm{O}\right)$ by the Gamma Ray Spectrometer (GRS) instrument suite indicates that the thickness of the desiccated layer cannot be larger than a few centimeters to tens of centimeters [Feldman et al., 2004].

[4] During winter, volatiles (essentially $\mathrm{CO}_{2}$ with $\mathrm{H}_{2} \mathrm{O}$ as a minor contaminant) condense on the surface. The seasonal volatile layer reaches a maximum extension up to $\sim 40^{\circ} \mathrm{S}$ between $\mathrm{Ls}=80^{\circ}$ and $\mathrm{Ls}=90^{\circ}$, as determined by thermal 
emission infrared spectroscopy [Giuranna et al., 2007]. The maximum observed thickness of this deposit estimated by laser altimetry is $0.9 \mathrm{~m}$ at $87^{\circ} \mathrm{S}$ and $\mathrm{Ls} \sim 155^{\circ}$ with a strong latitudinal gradient of up to $\sim 4 \mathrm{~cm}$ per latitude degree [Smith et al., 2001]. Gamma ray and neutron spectroscopy also permit the determination and mapping of $\mathrm{CO}_{2}$ ice surface column abundance with maximum values reaching $110 \mathrm{~g} / \mathrm{cm}^{2}$ at polar latitudes during early spring [Kelly et al., 2006; Litvak et al., 2007; Prettyman et al., 2009].

[5] In spring, after sunrise, the recession of the seasonal deposits can be monitored by visible imaging and nearinfrared spectroscopy. Around southern spring equinox $\left(\mathrm{Ls}=180^{\circ}\right)$, the layer of seasonal volatiles extends to $\sim 55^{\circ} \mathrm{S}$ [Fischbacher et al., 1969; James et al., 1979]. At large spatial scale, the evolution appears highly asymmetric, with the development of a so-called "cryptic region" [Kieffer et al., 2000] between longitudes $\sim 50^{\circ} \mathrm{E}$ and $\sim 230^{\circ} \mathrm{E}$ and after Ls $\sim 200^{\circ}$, characterized by a low albedo and a surface temperature corresponding to $\mathrm{CO}_{2}$ frost point as revealed by thermal infrared analyses. Kieffer et al. [2000] proposed that this cryptic region might be covered by a transparent slab of $\mathrm{CO}_{2}$ ice allowing the dark ground to be visible through it. However, near-infrared imaging spectroscopy reveals that the surface is highly contaminated by surface dust in the cryptic region and no clear $\mathrm{CO}_{2}$ ice has been unambiguously detected [Langevin et al., 2006, 2007; Titus et al., 2007; Brown et al., 2010]. Time-resolved maps built from the OMEGA data set [Langevin et al., 2007] also revealed the nonmonotonic behavior of both surface albedo and $\mathrm{CO}_{2}$ band strength. In the "anticryptic" region (i.e., the region that does not display the "cryptic" behavior) both these measures show first a strong increase during the first part of the recession (between $\mathrm{Ls} \sim 180^{\circ}$ and $250^{\circ}$ for latitudes around $80^{\circ} \mathrm{S}$ ), followed by a rapid decrease until the surface is free from volatiles. Finally, these data have permitted us to distinguish, for the first time, between the spatial and temporal behaviors of $\mathrm{CO}_{2}$ and $\mathrm{H}_{2} \mathrm{O}$.

[6] $\mathrm{H}_{2} \mathrm{O}$ ice is present over the entire seasonal deposits around $\mathrm{Ls} \sim 180^{\circ}-190^{\circ}$ as a surface contaminant of the $\mathrm{CO}_{2}$ ice, deposited here after sublimation from a large reservoir in Hellas basin [Langevin et al., 2007]. Surprisingly, this $\mathrm{H}_{2} \mathrm{O}$ ice disappears rapidly, whereas $\mathrm{CO}_{2}$ ice is still present on the surface. Recently, Brown et al. [2010] conducted a similar study using data obtained with the Compact Reconnaissance Imaging Spectrometer for Mars (CRISM) near-infrared spectrometer "mapping mode" [Murchie et al., 2007]. They also observe a strong temporal evolution of the $\mathrm{CO}_{2}$ band strength which they interpret in terms of evolution of ice grain size and $\mathrm{H}_{2} \mathrm{O}$ band strength. For the latter, they propose that the $\mathrm{H}_{2} \mathrm{O}$ ice could sublime and be the source of the simultaneous increase of $\mathrm{H}_{2} \mathrm{O}$ vapor in the atmosphere also observed by CRISM around Ls $=250^{\circ}$ [Smith et al., 2009].

[7] When observed at higher spatial resolution, terrain covered by seasonal volatiles shows dramatic temporal evolution during spring. Dark spots and fans lying on top of the bright seasonal deposits in early springtime were first observed by the Mars Orbiter Camera (MOC) [Malin et al., 1998]. The fan shape of some of these deposits leads to the idea that they are associated with jet-like activity [Kieffer, 2000, 2003; Piqueux et al., 2003]. Furthermore, retrieval of surface temperatures from the THEMIS thermal infrared camera shows that the dark features remain at the temper- ature of the sublimating $\mathrm{CO}_{2}$ ice, or less than $5 \mathrm{~K}$ above [Kieffer et al., 2006]. This indicates that the dark deposits are thin enough to be in thermal equilibrium with the underlying $\mathrm{CO}_{2}$-rich layer. Often, but not systematically, the dark fans are associated with radial features carved into the ground that have been informally named "spiders" [Piqueux et al., 2003]. Kieffer [2003], Piqueux et al. [2003], Kieffer et al. [2006], Kieffer [2007], Piqueux and Christensen [2008], Hansen et al. [2010], Portyankina et al. [2010a], Thomas et al. [2010, 2011], Pilorget et al. [2011] progressively detail various aspects of a plausible general scenario to account for the presence of these small-scale features and relate it to large spatial scale observations. The layer of $\mathrm{CO}_{2}$ ice can become transparent as the dust particles embedded in it migrate to the ground as a consequence of heating by solar radiation. Ice sublimation at the bottom of the $\mathrm{CO}_{2}$ slab results in a fast increase of pressure between the ice and the ground until the slab brakes at its weakest point, releasing a vent of $\mathrm{CO}_{2}$ gas and mobilizing material from the uppermost layers of the SPLD. From year to year, jet activity occurs at the same locations, progressively eroding the ground by carving meter-deep radial channels, which has been proposed as a scenario for creating araneiforms.

[8] Two remote sensing instruments on board the Mars Reconnaissance Orbiter (MRO) spacecraft are ideally suited to complement past observations of these processes and features by providing observations at an unprecedented spatial resolution: the High Resolution Imaging Science Experiment (HiRISE) visible camera [McEwen et al., 2007] and the CRISM visible and near-infrared imaging spectrometer [Murchie et al., 2007]. Hansen et al. [2010] and Thomas et al. [2010] describe the ground patterns and jetlike activity in the regions of Inca City as seen by HiRISE at a spatial resolution of $0.5 \mathrm{~m} /$ pixel. A new terminology is proposed by Hansen et al. [2010] to identify different types of "araneiform" features. Thomas et al. [2010] and Portyankina et al. [2010a] focus on the physical modeling of the jets and the cleaning and breaking processes inside the $\mathrm{CO}_{2}$ slab, respectively. First results from the analysis of high spatial resolution CRISM observations in four regions of the SPLD (Manhattan, Ithaca, Finger Lake and Giza) were reported by Titus et al. [2007]. They observed a strong correlation between the strength of the $\mathrm{CO}_{2}$ ice absorption bands and surface albedo that they interpret as evidence that dark areas are highly contaminated by mineral dust on the surface, which originates at least in part from the jets.

[9] In this paper, we aim to complement previous studies of the HiRISE data set [Hansen et al., 2010; Portyankina et al., 2010a; Thomas et al., 2010] by extending the number of regions of interest and by including spectral information from CRISM in our analyses. We have selected and studied 12 regions of interest in the south polar regions, based on the number and temporal resolution of available observations. We used data acquired by MRO during the 2007 southern Martian spring. Section 2 details how the data were processed for this study and provides estimates of the potential effect of atmospheric aerosols on the retrieval of surface parameters.

[10] Section 3 presents the results of the analyses of HiRISE and CRISM data sets. This section focuses on observational results and is clearly separated from section 4, where we present our hypotheses and scenarios to account 
Table 1. Geographic Coordinates of the 12 Regions of Interest Studied in This Paper

\begin{tabular}{lcc}
\hline \multicolumn{1}{c}{ Region Name } & Longitude & Latitude \\
\hline Agelenopsis & $87^{\circ} \mathrm{S}$ & $129^{\circ} \mathrm{E}$ \\
Clubonia & $86^{\circ} \mathrm{S}$ & $157^{\circ} \mathrm{E}$ \\
Dolomedes & $87^{\circ} \mathrm{S}$ & $175^{\circ} \mathrm{E}$ \\
Finger Lake & $84^{\circ} \mathrm{S}$ & $119^{\circ} \mathrm{E}$ \\
Giza & $84^{\circ} \mathrm{S}$ & $65^{\circ} \mathrm{E}$ \\
Inca City & $81^{\circ} \mathrm{S}$ & $63^{\circ} \mathrm{W}$ \\
Ithaca & $85^{\circ} \mathrm{S}$ & $178^{\circ} \mathrm{E}$ \\
Manhattan-Cicurina & $85^{\circ} \mathrm{S}$ & $102^{\circ} \mathrm{E}$ \\
Manhattan-Frontinella & $86^{\circ} \mathrm{S}$ & $97^{\circ} \mathrm{E}$ \\
Pirata & $77^{\circ} \mathrm{S}$ & $132^{\circ} \mathrm{E}$ \\
Richardson Crater & $73^{\circ} \mathrm{S}$ & $179^{\circ} \mathrm{E}$ \\
Starfish & $84^{\circ} \mathrm{S}$ & $118^{\circ} \mathrm{W}$ \\
\hline
\end{tabular}

for the various observations. From 10 of the 12 selected regions of interest, we derive time curves of surface albedo as well as $\mathrm{CO}_{2}$ ice and $\mathrm{H}_{2} \mathrm{O}$ ice spectral signatures from CRISM data over fixed areas. Time curves of surface reflectance in the red channel were also obtained from HiRISE in a similar way and consistency with CRISM is demonstrated. These time curves are completed by a series of CRISM visible RGB color composites and maps of $\mathrm{CO}_{2}$ and $\mathrm{H}_{2} \mathrm{O}$ band strength that reveal the spatial and temporal variability of these criteria at fine spatial scale. A series of color HiRISE images is then presented that illustrates the appearance and evolution of south polar terrain at submeter spatial resolution.

[11] Building upon the results presented in section 3, section 4 focuses on the physical processes responsible for the evolution of the appearance of polar terrain. In many previous spectroscopic studies of the south seasonal polar cap [e.g., Langevin et al., 2006, 2007; Brown et al., 2010], the interpretation of the spectra was based on physical modeling and spectral inversion to retrieve estimates of parameters such as ice particles grain size or amount of contaminants. However, because of the sensitivity of the reflectance spectra to a multitude of unconstrained parameters, inversion of the spectroscopic signal in terms of physical parameters is highly challenging and does not usually point to a unique solution in the case of layered semitransparent media as expected in this case [e.g., Douté and Schmitt, 1998]. We do not proceed here to a systematic physical inversion of the measured spectra. Our interpretation of the spectroscopic signals is partly based on discussions from the previously mentioned papers and relies extensively on the comparison with visual analyses of HiRISE color images acquired at the same time as CRISM data.

[12] It is important to keep in mind that the information retrieved from the analysis of visible and near-infrared images is only directly relevant for the uppermost layer of the seasonal deposits and ground, from the few first micrometers to centimeters depending on the transparency of the materials. Thus, the absence of detection of a spectral signature on the surface does not exclude the presence of the corresponding material, possibly as close as one millimeter below the surface. This is particularly important in this case where we observe a contamination of the uppermost layer of $\mathrm{CO}_{2}$ ice by mineral dust, sand and $\mathrm{H}_{2} \mathrm{O}$ ice. As the $\mathrm{H}_{2} \mathrm{O}$ ice is responsible for very strong absorptions in the near-IR, and minerals strongly absorb in the visible and
near-IR range, a low to moderate amount of these materials is enough to completely dominate the spectroscopic signal from the surface and hide the presence of underlying $\mathrm{CO}_{2}$.

\section{Data Sets and Methods}

\subsection{Regions of Interest}

[13] We have selected 12 regions of interest (ROIs) for this study (Table 1). The regions were chosen because of the large number of observations and high temporal resolution from both the HiRISE and the CRISM instruments. This study is mainly restricted to the southern spring observed by MRO in 2007. Whenever it is possible we have used the informal names attributed to these regions by the HiRISE team. When no name was yet attributed or when we decided to split an already named region into different parts, we chose names of spider species in reference to the observation of "araneiform" features in these areas. Note that all regions are located inside the SPLD unit but outside the bright permanent polar cap.

[14] From these 12 ROIs, eight are located inside the cryptic region as defined by [Kieffer et al., 2000], and four are located outside ("anticryptic region"): Inca City, Giza, Starfish and Richardson Crater. Note however, that the limits of the cryptic region are time dependent and that cryptic behavior can be observed at times outside the traditionally defined cryptic region [Kieffer et al., 2006; Langevin et al., 2007].

\subsection{HiRISE Data}

[15] We use both monochromatic HiRISE images obtained in the broad $0.55-0.85 \mu \mathrm{m}$ red band and HiRISE false color images produced from the near-infrared $(0.75-1.05 \mu \mathrm{m})$, red $(0.55-0.85 \mu \mathrm{m})$ and green-blue $(0.40-0.60 \mu \mathrm{m})$ channels (Figure 1a). Monochromatic images are used to retrieve absolute values of the reflectance factor at different times. We define here the reflectance factor as $\mathrm{I} /(\mathrm{F} \cos \mathrm{i})$, where I is the flux measured by the instrument $\left(\mathrm{W} \mathrm{m}^{-2} \mathrm{sr}^{-1}\right), \mathrm{F}$ is the incident solar flux $\left(\mathrm{W} \mathrm{m}^{-2} \mathrm{sr}^{-1}\right)$ and $\mathrm{i}$ is the incidence angle. With this definition, an ideal Lambertian surface would have a reflectance factor of 1 independently of the observation geometry. In the rest of this manuscript this unit will be referred to as "reflectance." Thus, because an accurate absolute calibration was crucial for this purpose, these images were entirely recalibrated from the raw data using an up-to-date version of the HiRISE calibration procedure. Because HiRISE color images are only used for morphologic studies and no absolute values are extracted from these data, it was not necessary to apply such a refined calibration procedure and standard PDS releases of JPG2000 color products available on the HiRISE web server (http:// hirise.lpl.arizona.edu/) were used.

\subsection{CRISM Data}

[16] Both visible and near-infrared CRISM data are used in this study. We only used hyperspectral targeted observations at full spectral resolution: full resolution targeted measurement (FRT), half resolution short targeted measurement (HRS) and half resolution long targeted measurement (HRL) products. Results from analyses of CRISM data acquired in the complementary "mapping mode" (reduced spatial and spectral resolutions) have 
a)

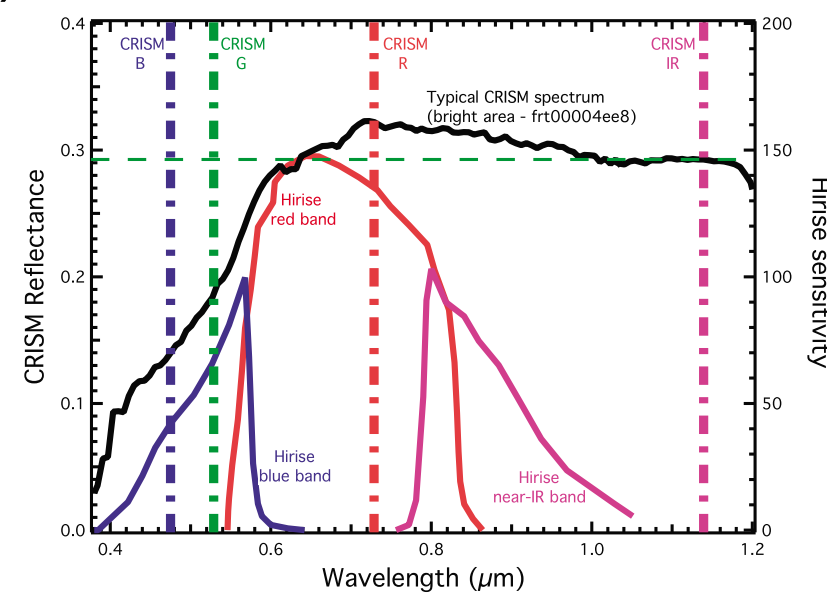

C)

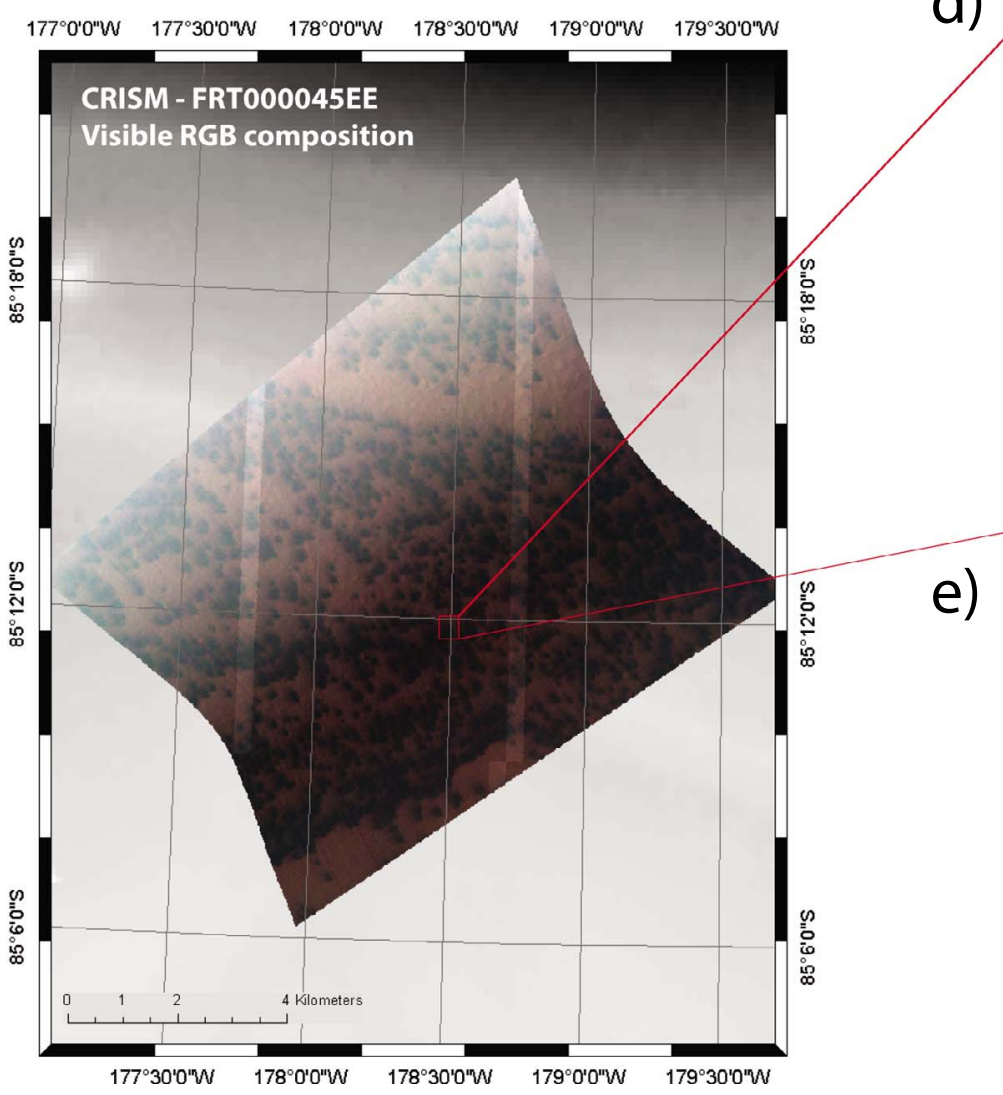

e) b)

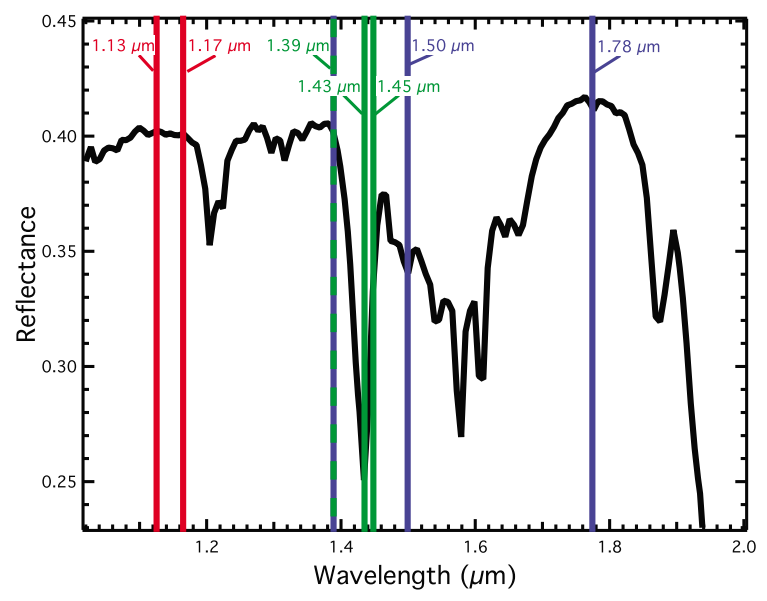

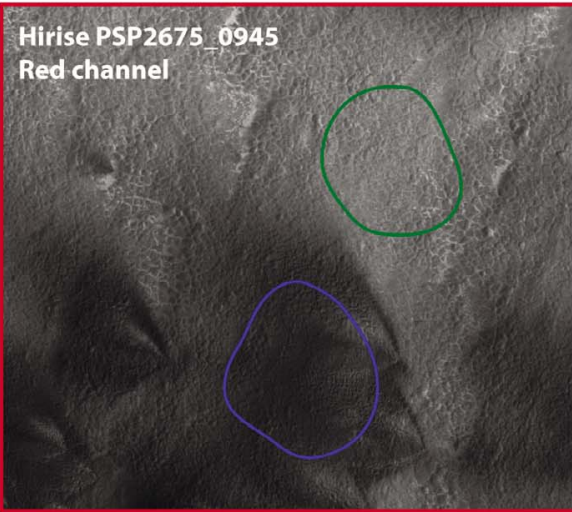

Figure 1. Comparison between the spectral and spatial ranges and resolutions of the HiRISE and CRISM instruments. (a) A typical visible reflectance spectrum of the dusty $\mathrm{CO}_{2}$ ice layer that covers part of the south polar area during Martian spring measured by CRISM (region of Ithaca) is plotted in black. The level of sensitivity of HiRISE in the three "color" channels is represented [from Delamere et al., 2010] as well as the particular wavelength bands used to display the CRISM false color images (CRISM R, G and B) and to evaluate the level of reflectance in the continuum of the spectrum (CRISM IR). (b) Part of a typical near-IR CRISM spectrum of the dusty $\mathrm{CO}_{2}$ layer over the spectral range 1.0 $2.0 \mu \mathrm{m}$ and identification of the spectral channels used for the calculations of band strength criteria for the $\mathrm{CO}_{2}$ and $\mathrm{H}_{2} \mathrm{O}$ ices according to the definitions proposed by Langevin et al. [2007]. (c, d, e) Examples of definitions of a bright and a dark area for a HiRISE and a CRISM observation in the region of Ithaca at $\mathrm{Ls}=187.5^{\circ}$. The spatial definition of the HiRISE observation is $0.5 \mathrm{~m} /$ pixel, whereas the spatial definition of the CRISM observation is $19 \mathrm{~m} /$ pixel. Despite its lower resolution, CRISM is still able to sufficiently resolve the dark fans and the bright soil. 
already been published by Brown et al. [2010]. Radiometric and spectral calibration was performed using the CAT software released by the CRISM team [Morgan et al., 2009]. We systematically applied the division by the cosine of the incidence angle as provided by derived data records (DDR) auxiliary products to retrieve the reflectance factor $\mathrm{I} /(\mathrm{F} \cos \mathrm{i})$ consistent with HiRISE units.

[17] We build color composite RGB images from the CRISM visible hyperspectral cubes by selecting the following three individual spectral bands: $\mathrm{R}=0.73 \mu \mathrm{m}, \mathrm{G}=$ $0.52 \mu \mathrm{m}$ and $\mathrm{B}=0.47 \mu \mathrm{m}$ (Figure $1 \mathrm{~b}$ ). From near-infrared hyperspectral cubes, we calculated three spectral criteria: reflectance in the continuum at $1.14 \mu \mathrm{m}$, strength of the $1.4 \mu \mathrm{m} \mathrm{CO}_{2}$ absorption band and strength of the $1.5 \mu \mathrm{m} \mathrm{H}_{2} \mathrm{O}$ absorption band. All calculations were made according to the definitions proposed by Langevin et al. [2007] and subsequently reused by Brown et al. [2010] with CRISM mapping mode data.

[18] In a next step, CRISM visible color composites and maps of $\mathrm{CO}_{2}$ and $\mathrm{H}_{2} \mathrm{O}$ band strength were projected in a south polar stereographic projection, consistent with HiRISE images and other Martian data sets. All maps were finally imported into a GIS software for display as well as further multiinstruments spatial analyses. For display, constant stretches were usually applied to all CRISM maps in order to allow direct comparisons between different times and different areas.

\subsection{Temporal Evolution of "Regions of Interest" Observed by HiRISE and CRISM}

[19] We extracted the absolute values of the three different near-IR spectral criteria (CRISM) or visible surface reflectance (HiRISE) at fixed locations in the different regions of interest. This allows us to follow their evolution as a function of time. The selection of these locations was essentially driven by the necessity to remain as close as possible to the center of the CRISM images to limit the strong photometric artifacts that affect these data [Murchie et al., 2007]. In each region, we systematically extracted values from a "dark area" and from a "bright area" close to each other. An example of the definitions of the ROIs in Ithaca is provided in Figures 1c, 1d, and 1e.

\subsection{Modeling of the Impact of Aerosols on Retrieved Surface Reflectance Values}

[20] The Martian atmosphere is always dusty, with variable amount of mineral aerosols depending upon time and location [Lemmon et al., 2004; Smith, 2004]. Because the values of surface reflectance measured by HiRISE and CRISM are affected by the atmospheric aerosols content, it is necessary to estimate the resulting uncertainty on these values. The Monte Carlo model developed by Vincendon et al. [2007] was used in combination with values of atmospheric opacities, $\tau$, measured by the OMEGA instrument over the South Pole [Vincendon et al., 2008, 2009] simultaneously with HiRISE and CRISM measurements.

[21] The values of surface reflectance that we measured with HiRISE for this study range between 0.2 and 0.6. Atmospheric opacities at the times of the measurements are typically in the range 0.4 and 1.0. Under these conditions, relative errors on the retrieval of surface reflectance can reach $10 \%$ for bright surfaces and as much as $30 \%$ for very dark surfaces [Portyankina et al., 2010b]. These uncertainties are significant, but most of the time and for most of terrain studied, they remain an order of magnitude below the temporal variations of reflectance over the entire spring season.

[22] To get a clear view of the potential effect of aerosols on interpretation of temporal variations of reflectance, we applied a correction of their effects on HiRISE data [Portyankina et al., 2010b], using a method adapted from Vincendon et al. [2007]. Figure 2 shows time curves of reflectance in the HiRISE red band for both bright and dark areas in the region of Ithaca and Inca city, before and after correction of the aerosols influence, as well as the temporal evolution of $\tau$ retrieved from OMEGA data. These values of $\tau$ were measured above the permanent polar cap $\left(87^{\circ} \mathrm{S}\right.$, $30^{\circ} \mathrm{W}$ ) and vertically extrapolated to the altitude of the regions of interest. As a consequence, possible spatial variations of the atmospheric opacity over the area covered by seasonal volatiles are not considered. The results clearly show that the effects of aerosols are noticeable but do not modify the general trends in a significant way. Therefore, we decided not to apply any systematic correction of the effects of aerosols to the data for the rest of this study. Note that all maps and curves presented by Langevin et al. [2007] and Brown et al. [2010] are also built without any correction of the aerosol effects. The temporal evolution of both surface reflectance and atmospheric opacity displayed in Figure 2 are discussed in detail in section 4.3.

\section{Observations}

[23] Figure 3 shows the temporal evolution of continuum reflectance as well as $\mathrm{CO}_{2}$ and $\mathrm{H}_{2} \mathrm{O}$ band strength, all retrieved from CRISM data, between the beginning of spring and summer. Both generally consistent behavior and some spatial variability are evident from these curves. In Figure 4, we propose a schematic view of the typical behavior and define points on the curves that will be used in the rest of the discussion.

[24] For continuum reflectance and $\mathrm{CO}_{2}$ band strength, we identify three phases (Figures $4 \mathrm{a}$ and $4 \mathrm{~b}$ ). The first phase, between points $\mathrm{A}$ and $\mathrm{B}$, consists in a decrease of continuum reflectance and $\mathrm{CO}_{2}$ band strength. Later, between points $\mathrm{B}$ and $\mathrm{C}$, both criteria show a significant increase. Finally, between points $\mathrm{C}$ and $\mathrm{D}$, both criteria decrease again until the end of sublimation of volatiles. We observe a systematic shift of the position of $\mathrm{B}$ between continuum reflectance and $\mathrm{CO}_{2}$ band strength, this point being always encountered later in the case of $\mathrm{CO}_{2}$. On the other hand, point $\mathrm{C}$ occurs approximately at the same time for both criteria. A significant spatial variability is also observed for the positions of both $\mathrm{B}$ and $\mathrm{C}$ points. This appears to be related to variations with latitude and asymmetry of the retreat of seasonal volatiles layer. Unfortunately, the global dust storm in "Martian year" (MY) 28 prevented CRISM and HiRISE observations of the surface between Ls $=260^{\circ}$ and Ls $=290^{\circ}$ in most areas. Thus, the exact time of the end of sublimation of volatiles, point $\mathrm{D}$, cannot be accurately constrained from our data.

[25] The behavior of $\mathrm{H}_{2} \mathrm{O}$ band strength is very different from $\mathrm{CO}_{2}$ (Figure 4c): we observe either a monotonic decrease of this value or a plateau, until point $\mathrm{E}$, and then a decrease. The $\mathrm{H}_{2} \mathrm{O}$ band strength always reaches a value of 


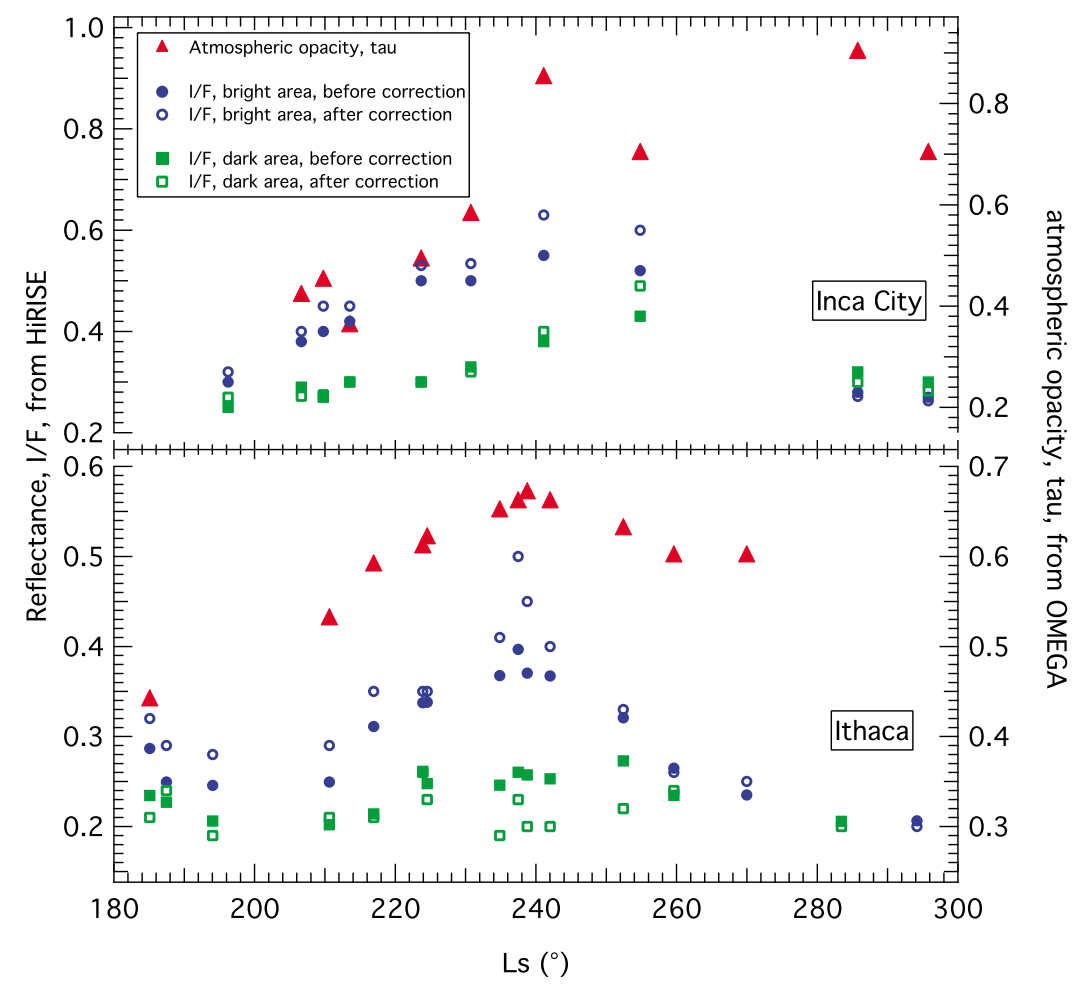

Figure 2. Examples of the temporal evolutions of surface reflectance of bright and dark areas determined by HiRISE (red channel, see Figure 1) in the regions of Ithaca and Inca City. Values of the atmospheric opacity tau, extrapolated from OMEGA measurements above the south permanent polar cap, are also displayed. In both regions, we identify an interesting and puzzling positive correlation between the temporal evolutions of surface reflectance and atmospheric opacity. By modeling the effects of aerosols on the retrieval of surface reflectance, we corrected their influence on reflectance values measured by HiRISE for both bright regions and dark fans. Comparison of uncorrected and corrected data shows that when the effect of aerosols is not negligible, it essentially results in a global shift of the curves but does not modify the observed general trends.

0 before $\mathrm{CO}_{2}$ band strength. This does not necessarily indicate that $\mathrm{H}_{2} \mathrm{O}$ ice entirely disappears from the surface. Because water ice is a relatively minor compound in the volatile layer, the early disappearance of $\mathrm{H}_{2} \mathrm{O}$ spectral signature might simply indicate that concentration of $\mathrm{H}_{2} \mathrm{O}$ ice decreases below its detection threshold but is still present in low amount. However, because $\mathrm{H}_{2} \mathrm{O}$ shows extremely strong absorptions in the near-infrared, this detection threshold will be very low [see Langevin et al., 2007] and an absence of detection is nearly synonymous to a physical absence.

[26] A comparison of the behavior of dark and bright areas shows parallel temporal evolution of the two types of terrain. Note that the difference in albedo between bright and dark areas is usually much smaller than the amplitude of temporal albedo changes during spring. We also usually observe a decrease of the contrast between bright and dark areas but the time at which this occurs appears to be variable from one area to another.

[27] Figure 5 presents two examples of comparisons between the temporal evolution of surface reflectance of fixed regions of interest seen by HiRISE and CRISM, respectively. A significant proportion of the data for these two regions were acquired simultaneously by both instruments. Therefore, solar illumination and atmospheric conditions were similar. Furthermore, both instruments acquired data at low emission angle, close to nadir. In both regions, Inca City and Ithaca, observations by the two instruments show good agreement with few differences despite the difference in spatial resolution (Figures $1 \mathrm{~d}$ and 1e). A general shift of reflectance factor of $10-15 \%$ is observed, HiRISE showing systematically lower values than CRISM. The difference of channel wavelengths (Figures 1a and 1b) cannot explain this systematic shift. However, the observed difference is consistent with the $10 \%$ uncertainty in the absolute calibration of the two instruments [McEwen et al., 2007; Murchie et al., 2007] and does not affect our interpretation in a significant way. The year-to-year reproducibility of these observations was also tested using HiRISE data in the regions of Inca City and Ithaca and was judged to be excellent.

[28] Figures 6 and 7 show two examples of time series of CRISM observations: RGB color composites at visible wavelengths, maps of $\mathrm{CO}_{2}$ band strength and maps of $\mathrm{H}_{2} \mathrm{O}$ band strength for the areas of Ithaca and Giza, respectively. Not all analyzed images are displayed in Figures 6 and 7. Complete time series for these two regions as well as similar figures for six other regions are available in the auxiliary material (Figure S1). ${ }^{1}$ Both $\mathrm{CO}_{2}$ and $\mathrm{H}_{2} \mathrm{O}$ band strengths

\footnotetext{
${ }^{1}$ Auxiliary materials are available in the HTML. doi:10.1029/ 2010JE003790
} 
a. Reflectance at $1.14 \mu \mathrm{m}$
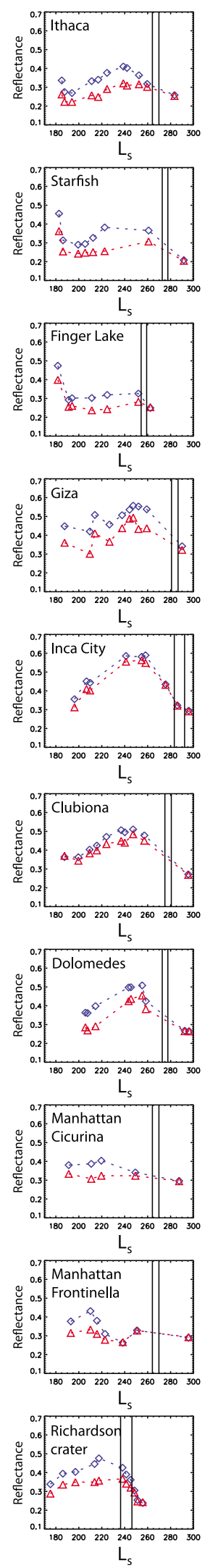

b. $\mathrm{CO}_{2}$ band strength
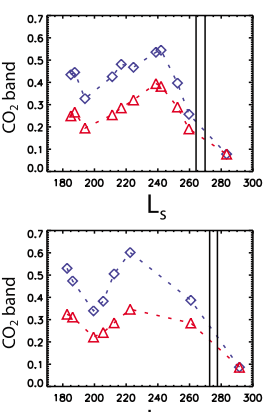

$\mathrm{L}_{\mathrm{s}}$
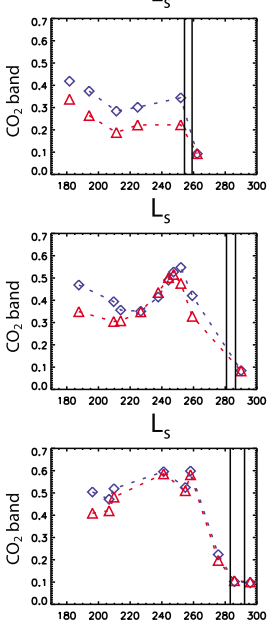

$\mathrm{L}_{\mathrm{s}}$
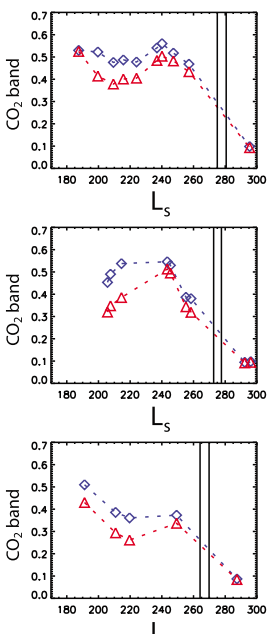

4

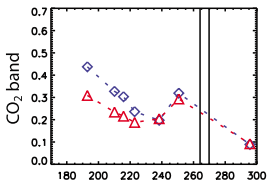

$\mathrm{L}_{\mathrm{s}}$

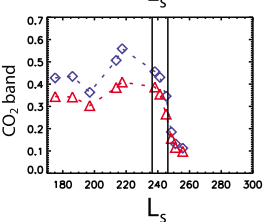

C. $\mathrm{H}_{2} \mathrm{O}$ band strength
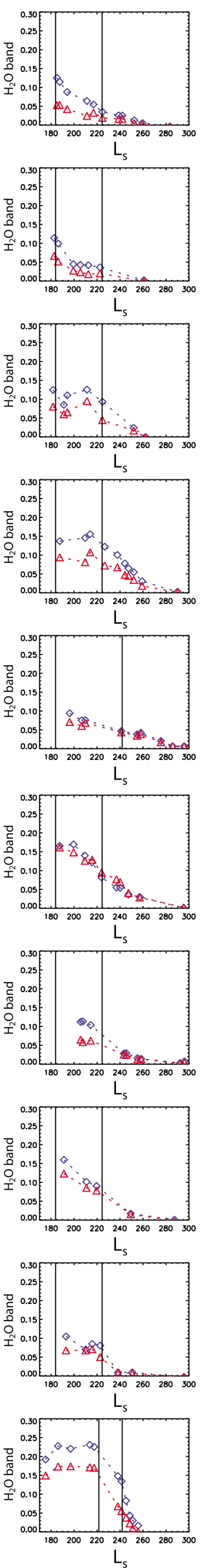

are calculated in the same way as for Figure 3 and displayed over the same ranges of values. In a similar way, RGB color composites are displayed with constant color stretches for all areas and times, allowing direct comparison.

[29] In addition to the general behavior already described from the curves displayed in Figures 3 and 4, the time series of CRISM maps permit interesting observations of the spatial variability of evolution of terrain at small spatial scale. In particular, a positive correlation between visible albedo and strength of the $\mathrm{CO}_{2}$ band strength stands out: bright terrain presents the highest values of $\mathrm{CO}_{2}$ band strength and dark deposits display very weak, or even no, signature of $\mathrm{CO}_{2}$ ice, even in early spring. Water ice also shows some spatial correlation with albedo and $\mathrm{CO}_{2}$ ice in early spring: dark deposits show no signature of water ice and the highest values of $\mathrm{H}_{2} \mathrm{O}$ band strength usually correspond to the highest values of $\mathrm{CO}_{2}$ band strength. However, this spatial correlation disappears later in spring as $\mathrm{H}_{2} \mathrm{O}$ band strength decreases during the brightening of the volatile layer whereas $\mathrm{CO}_{2}$ band strength increases. This is particularly obvious in Giza where bright areas corresponding to high $\mathrm{CO}_{2}$ band strength and low $\mathrm{H}_{2} \mathrm{O}$ band strength appear at Ls $=209.6^{\circ}$ (Figure 7).

[30] In order to interpret the temporal evolution, we visually analyzed the high-resolution color images obtained by HiRISE within our different regions of interest with a special emphasis on images taken at particularly interesting times.

[31] Figure 8 presents six images extracted from HiRISE observations before Ls $=200^{\circ}$ (phase $\mathrm{A} \rightarrow \mathrm{B}$ of the time curves in Figures 3 and 4). All images show dark fans lying on top of brighter surfaces. Comparison between the colors seen on HiRISE images and spectral information from CRISM indicates that what we see as a brighter ground around dust fans is the surface of the volatile layer, contaminated by some fine mineral dust. Fans appear darker because they consist of larger mineral grains that settle close to the source of the jet. On the other hand, smaller and redder grains emitted by the same process are widespread

Figure 3. Temporal evolutions of different spectral criteria calculated from CRISM observations of 10 selected regions. These spectral criteria are designed to provide good proxies for (a) surface albedo, (b) strength of the $\mathrm{CO}_{2}$ ice absorption and (c) strength of the $\mathrm{H}_{2} \mathrm{O}$ ice absorption (see section 2). For each region of interest, the spectral criteria are calculated for both a fixed homogeneous dark region (blue diamonds) and a fixed homogeneous bright region (red triangles). The shapes of these curves are discussed in more detail in Figure 4. In addition to the CRISM time curves, the range of Ls where OMEGA sees the volatiles disappear (i.e., crocus dates) from the surface is represented between two vertical black lines [from Langevin et al., 2007]. These OMEGA limits are represented for $\mathrm{CO}_{2}$ ice in Figures 3a and $3 \mathrm{~b}$ and for $\mathrm{H}_{2} \mathrm{O}$ ice in Figure $3 \mathrm{c}$. The exact timing of the disappearance of $\mathrm{CO}_{2}$ ice is difficult to establish from CRISM MY 28 data because of the dust storm that prevented good observations of the surface between $\mathrm{Ls}=260^{\circ}$ and $280^{\circ}$. For $\mathrm{H}_{2} \mathrm{O}$ ice, we observe discrepancies between results obtained form OMEGA MY 27 data and CRISM MY 28 data that are discussed in section 4.3. 

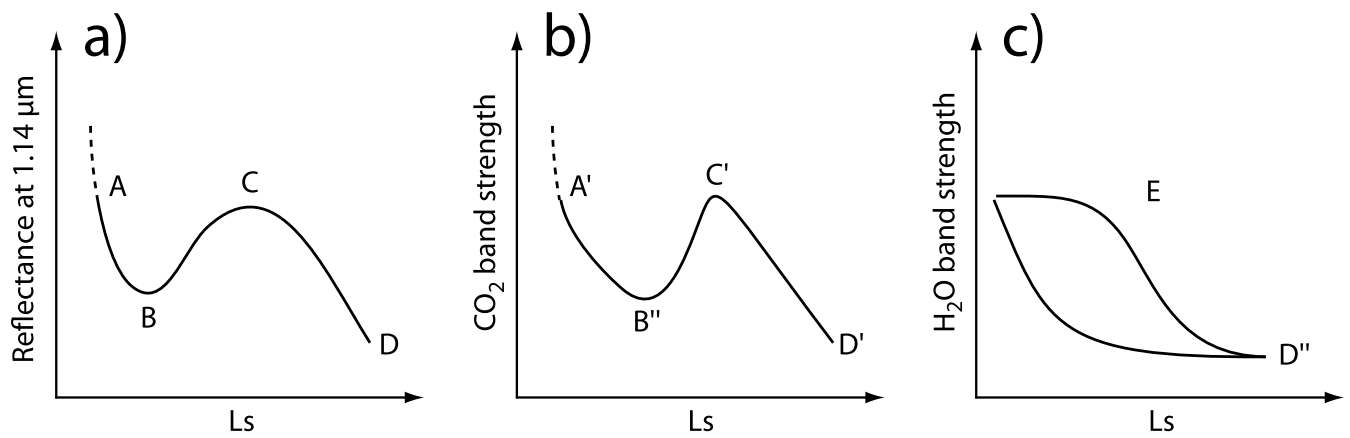

Figure 4. Schematics of the main trends of the three spectral criteria as observed for most of the regions of interest in Figure 3. The trends for (a) continuum reflectance and (b) $\mathrm{CO}_{2}$ are labeled with letters used to discriminate different phases of the curves and reference to them in the text. The evolutions of continuum reflectance and $\mathrm{CO}_{2}$ band strength show generally similar behaviors. However, we note a quasisystematic shift of the $\mathrm{B}$ point toward a later time for $\mathrm{CO}_{2}$ band strength compared to albedo. For most of the regions, the relative decrease between points $\mathrm{A}$ and $\mathrm{B}$ is more significant for the $\mathrm{CO}_{2}$ band strength than for the albedo. Finally, $\mathrm{CO}_{2}$ band depth seems to display a "sharper" transition around point $\mathrm{C}$. Despite a generally similar shape, there is also significant variability from one region to another (see section 4). (c) In contrast, the behavior of the band strength of $\mathrm{H}_{2} \mathrm{O}$ ice is different: we observe either a continuous decrease or a plateau until point $\mathrm{E}$ and then a decrease. In every case we studied, the $\mathrm{H}_{2} \mathrm{O}$ ice spectral signature disappears from the surface before the $\mathrm{CO}_{2}$ ice spectral signature.

around the surrounding area, eventually together with atmospheric deposits. In the rest of the manuscript, we will refer to the large grains in the dark fans as "sand" and to the small reddish grains as "dust." In addition to a difference of particle size, these two classes probably also present a difference of mineralogical composition, the sand-sized dark grains being likely basaltic and the fine red dust being rich in iron oxides. On all these images, we also observe very bright/white features, usually close to the dark fans. Often, these features are elongated, corresponding to bright channels carved in the ground, part of isolated araneiform features or of dense channels networks (see discussion of different araneiform features by Hansen et al. [2010]). After careful analysis of the images, we excluded the possibility that bright features could be caused by specular reflections or another photometric effect because of the wide range of orientations compared to the direction of the Sun at the time of HiRISE measurements (represented in the diagrams on the right of each HiRISE picture, see Figure 8 caption).

[32] Figure 9 shows a compilation of HiRISE images acquired around the time of the transition between the darkening and brightening phase on the time curves (Figures 3 and 4, point B). At this time, we observe "haloes" with a characteristic blue color around the external edges of the dark surface deposits in most of studied areas. As wavelength bands used for HiRISE and CRISM color images are different, haloes do not appear blue anymore on CRISM images but show light gray to white colors (not shown). However, for consistency with the previous description, we will continue to refer to them as "blue haloes" for the rest of this paper. In Ithaca and Starfish, the contrast of colors between haloes (light gray) and bright icy soil (red) is very significant. In comparison, only a very small contrast is observed between these two types of terrains on the $\mathrm{CO}_{2}$ band strength maps, blue haloes displaying an intermediate value of $\mathrm{CO}_{2}$ band strength between dark deposits and bright icy soil. $\mathrm{H}_{2} \mathrm{O}$ band strength is also very low inside blue haloes. This is especially obvious in Giza where both dark and bright/blue deposits can present values of $\mathrm{H}_{2} \mathrm{O}$ band strength close to 0 .

[33] Figure 10 shows the comparison of visible (Figure 10, left) and near-infrared (Figure 10, right) spectra recorded by CRISM over the three different types of terrains in the regions of Ithaca (Figures 10a and 10b) and Giza (Figures 10c and 10d). In the visible spectral range, CRISM

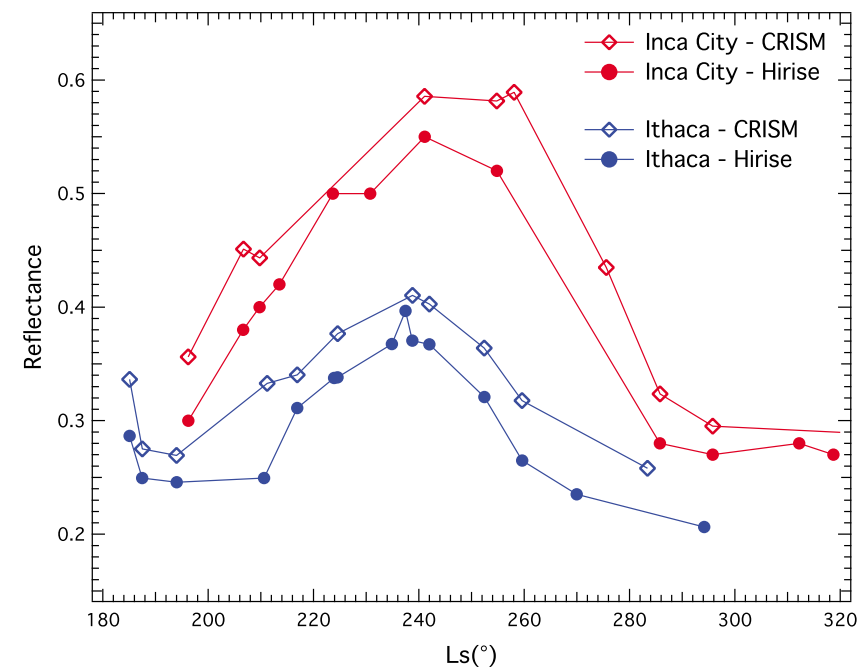

Figure 5. Temporal evolution of surface reflectance measured by HiRISE in the wide red visible channel compared to surface reflectance measured by CRISM at $1.14 \mu \mathrm{m}$ for bright soil in the regions of Ithaca (blue curves, in the cryptic region) and Inca City (red curves, outside the cryptic region). The general relative trends are very similar in both regions. The systematic absolute shift between the two instruments is attributed to uncertainties in absolute calibration. 
RBG colors
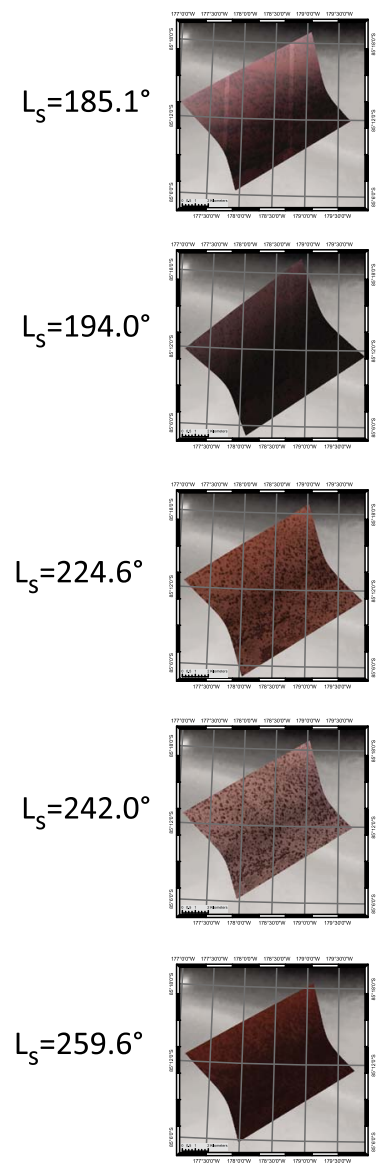

$\mathrm{CO}_{2}$ band
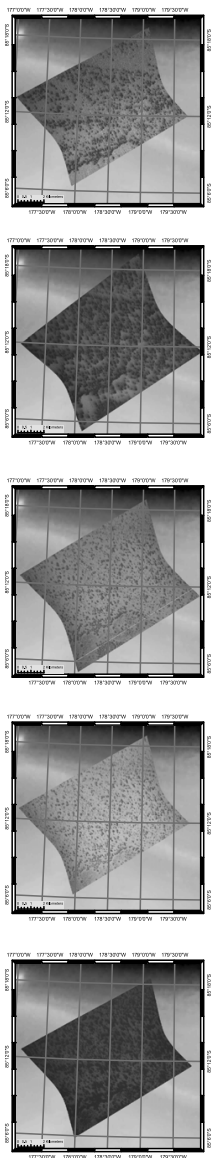

$\mathrm{H}_{2} \mathrm{O}$ band
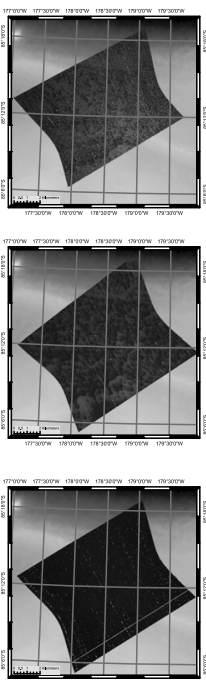

Figure 6. Temporal evolution of the region of Ithaca as seen through successive selected CRISM visible and nearinfrared observations. (left) RGB color composite: $\mathrm{R}=$ $0.73 \mu \mathrm{m}, \mathrm{G}=0.52 \mu \mathrm{m}, \mathrm{B}=0.47 \mu \mathrm{m}$ (see Figure 1 ). The color rendering (stretching of each channel) is kept constant for all images at different times and in different regions to allow for direct comparisons of overall albedo and color: $\mathrm{R}=0.23-0.77, \mathrm{G}=0.14-0.69$, and $\mathrm{B}=0.12-0.60$. (middle) $\mathrm{CO}_{2}$ band strength, linearly stretched between 0 (black) and 0.7 (white). (right) $\mathrm{H}_{2} \mathrm{O}$ band strength, linearly stretched between 0 (black) and 0.3 (white). Stretches for $\mathrm{CO}_{2}$ and $\mathrm{H}_{2} \mathrm{O}$ band strength maps are identical to the $y$ axis ranges of all plots in Figure 3. A more complete version is also provided in the auxiliary material (Figure S7).

spectra confirm that the reflectance of the blue haloes is larger at blue wavelengths but lower at red wavelengths and in the near-IR continuum compared to the surrounding bright soil. At longer wavelengths, band strengths of both $\mathrm{CO}_{2}$ and $\mathrm{H}_{2} \mathrm{O}$ are usually reduced in blue haloes. It is interesting to note the difference between the regions of Ithaca and Giza. In Giza, both the strength of the $\mathrm{CO}_{2}$ bands and near-IR continuum are just a bit lower in blue haloes than in bright soils. In Ithaca, the contrast of both continuum reflectance and band strength is far more prominent. The $2.7-3.7 \mu \mathrm{m}$ range also shows strong spatial and temporal variability with, in particular, the spectra of blue haloes in the region of Giza that show a significant level of reflectance between 2.9 and
$3.3 \mu \mathrm{m}$ whereas this region of the spectrum shows saturated absorption on all other spectra.

[34] Figure 11 shows the temporal evolution of a fixed scene in the region of Ithaca between Ls $=185.1^{\circ}$ and $\mathrm{Ls}=$ $210.6^{\circ}$. Both dark surface deposits and white elongated features previously described are present in the scene, surrounded by intermediate-albedo soil. Between Figures 11a and $11 \mathrm{~b}\left(\mathrm{Ls}=185.1^{\circ}\right.$ and $\left.\mathrm{Ls}=187.6^{\circ}\right)$, a significant increase of the area that is covered by dark fans is obvious, either as a result of movements of mineral grains driven by surface wind or as a result of repeated emissions of dark grains from the jet sources. Note in particular that the bright/ white feature close to the top left corner has been entirely covered by dark material. Another prominent change is the appearance of relatively blue areas within the dark fans, either on their external edges or in the middle. Figure $11 \mathrm{c}$ $\left(\mathrm{Ls}=194.0^{\circ}\right)$ shows even more drastic changes: significant fraction of areas previously covered by dark deposits now shows a distinct blue color whereas the center parts of the dark fans are uniformly black, corresponding to the apparition of "blue haloes" as described in Figures 9 and 10. The two small dark fans on the right edge of the image are entirely blue, but a significant contrast of darkness with surrounding areas is still observed. Finally, the blue entirely

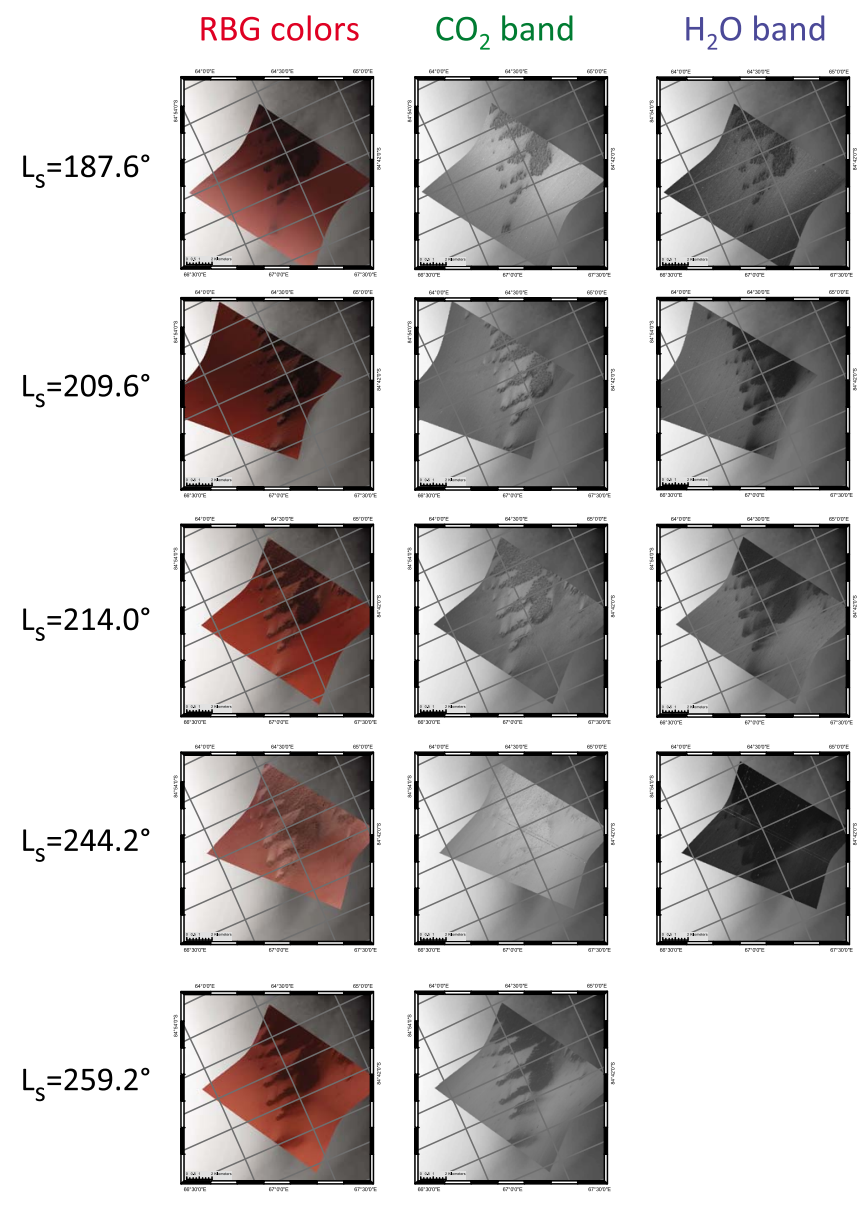

Figure 7. Same as Figure 6 except for the region of Giza. A more complete version of this figure is also provided in the auxiliary material (Figure S5). 

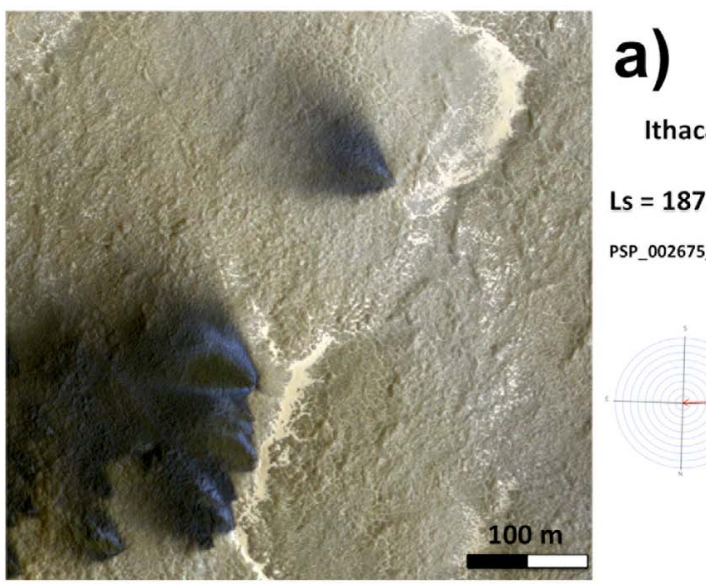

Ithaca

Ls $=187.46^{\circ}$

PSP_002675_0945
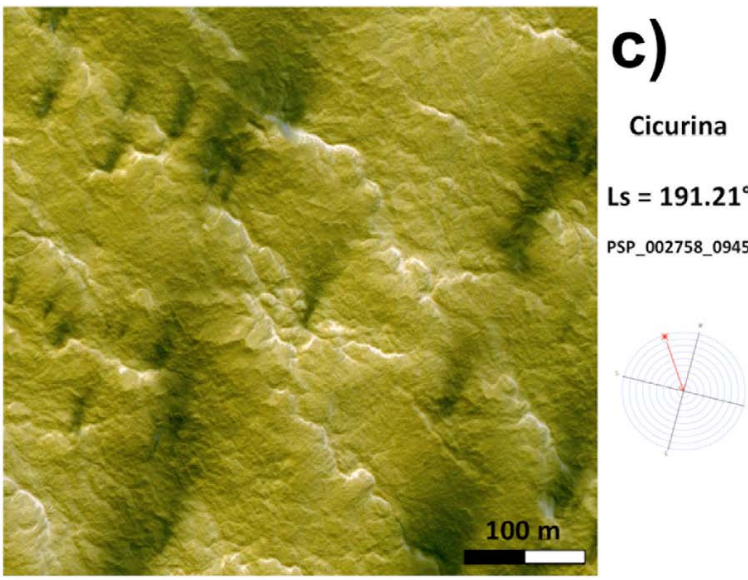

$L s=191.21^{\circ}$

PSP_002758_0945

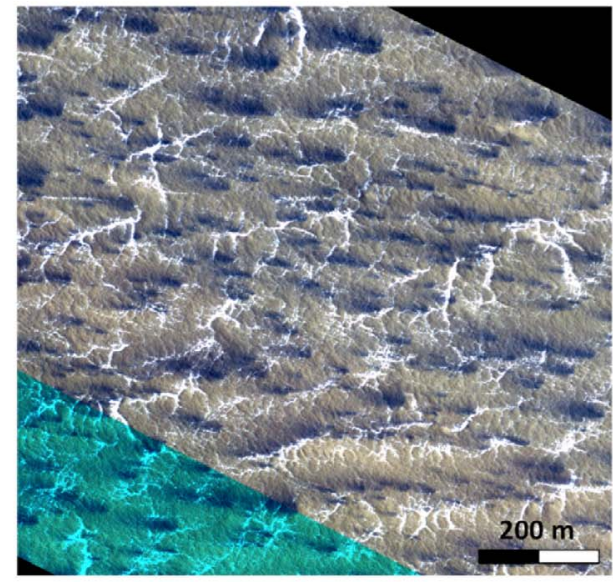

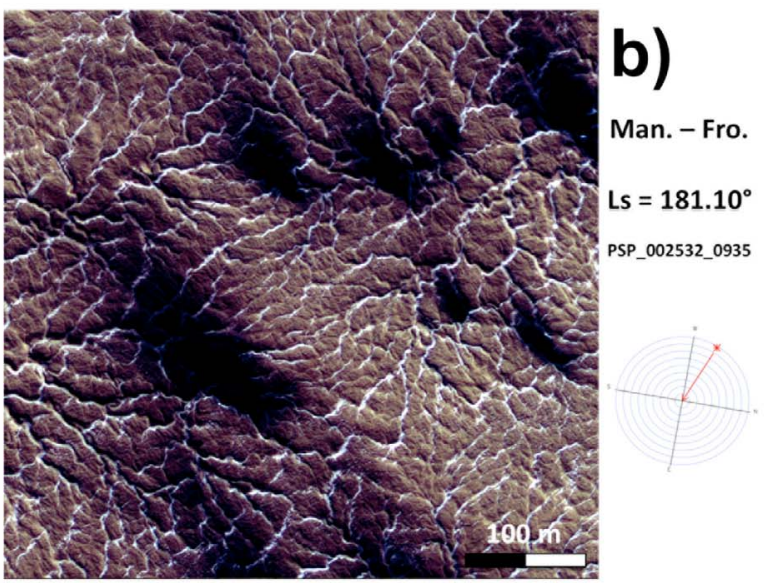
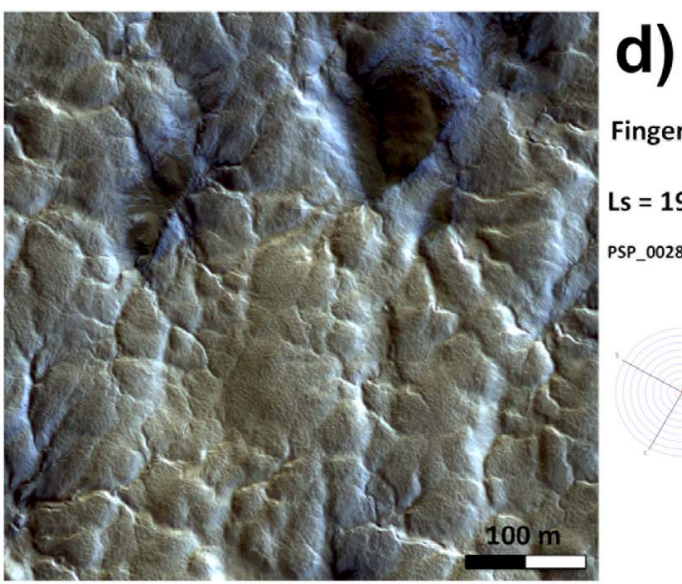

Finger Lake

$L s=194.12^{\circ}$

PSP_002822_0955

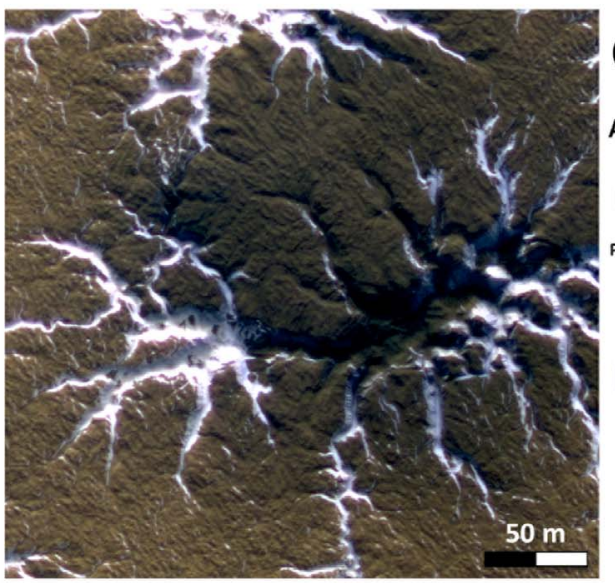

e)

Agelenopsis

Ls $=206.4^{\circ}$ PSP_003087_0930

Figure 8. Examples of "fresh" dark fans observed by HiRISE between Ls $=175^{\circ}$ and $206^{\circ}$ in different regions. All images also show examples of "bright features" that we interpret as clean slopes on various types of small-scale topography. The sources of the dark fans are usually observed close to the bright features, enforcing the idea that they are associated in some way. The possible link between these two types of features is further discussed in section 4.1. The geographic orientation of the image as well as the position of the Sun in the sky is represented on the diagram on the right of each HiRISE image. The Sun is represented by the small red star. Incidence angle is represented by the distance between the Sun and the center of the diagram: it is $0^{\circ}$ in the center of the diagram and $90^{\circ}$ on the outer circle. Concentric circles correspond to isoincidence angles by steps of $10^{\circ}$. Similar diagrams are shown for all HiRISE images in Figures 9, 11 and 12. 

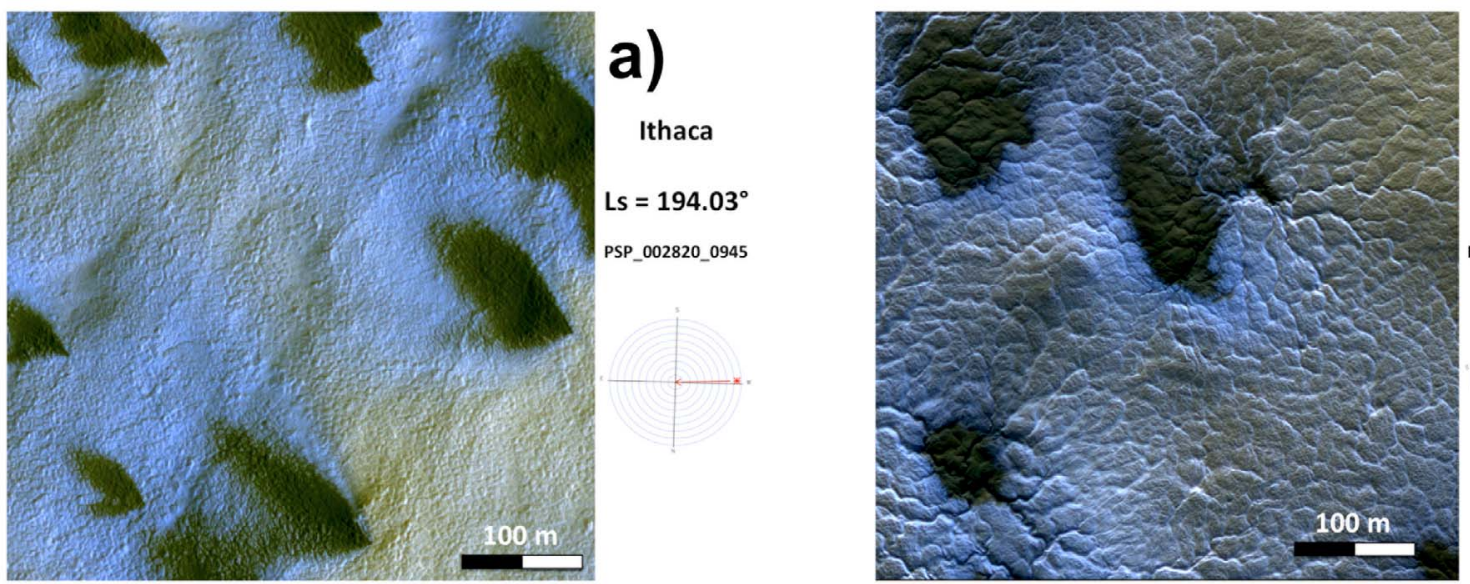

\section{b)}

$L s=194.03^{\circ}$

PSP_002820_0945
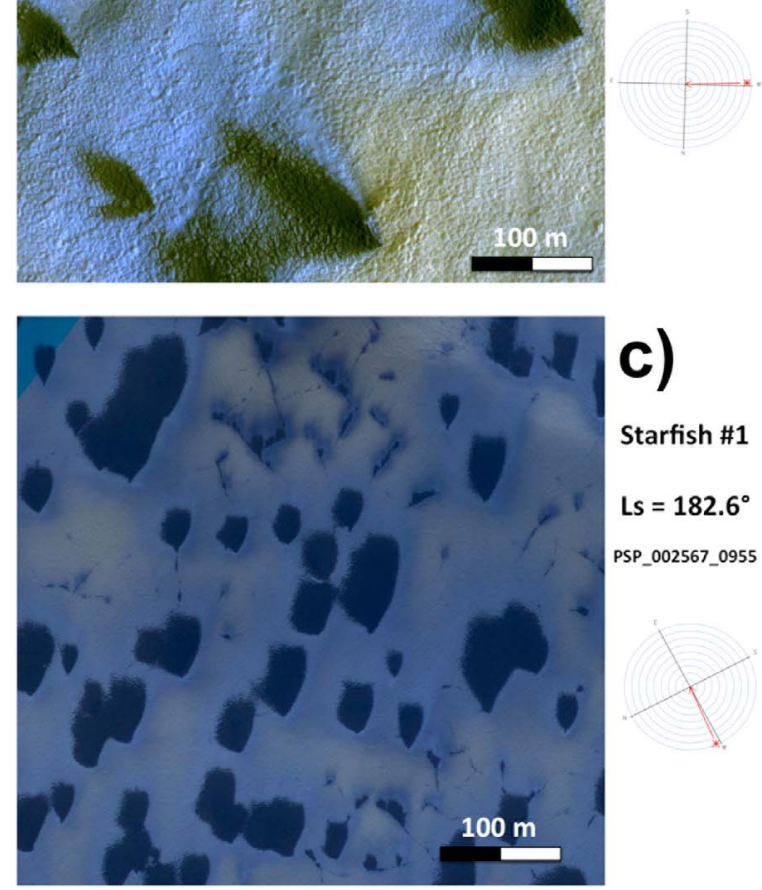

\section{c)}

Starfish \#1

$L S=182.6^{\circ}$

PSP_002567_0955

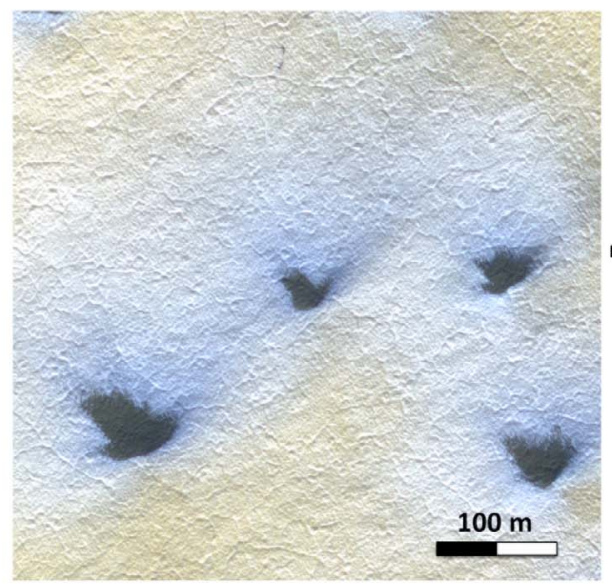

\section{d)}

Starfish \#2

$L S=182.6^{\circ}$

PSP_002567_0955

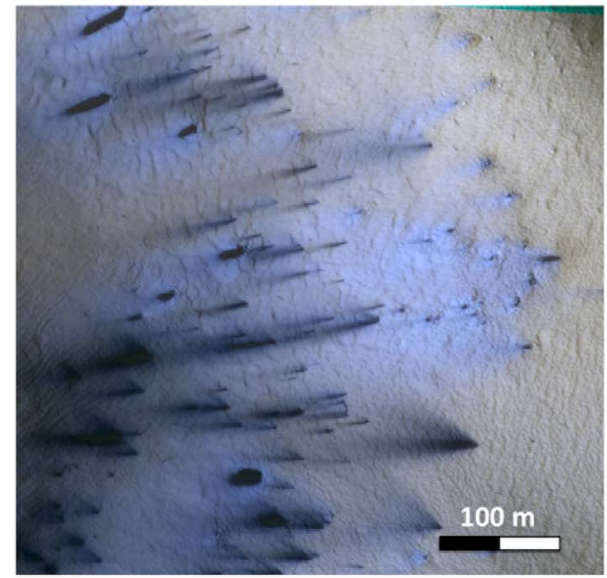

\section{d) \\ Inca City}

$L S=196.2^{\circ}$

PSP_002868_0985

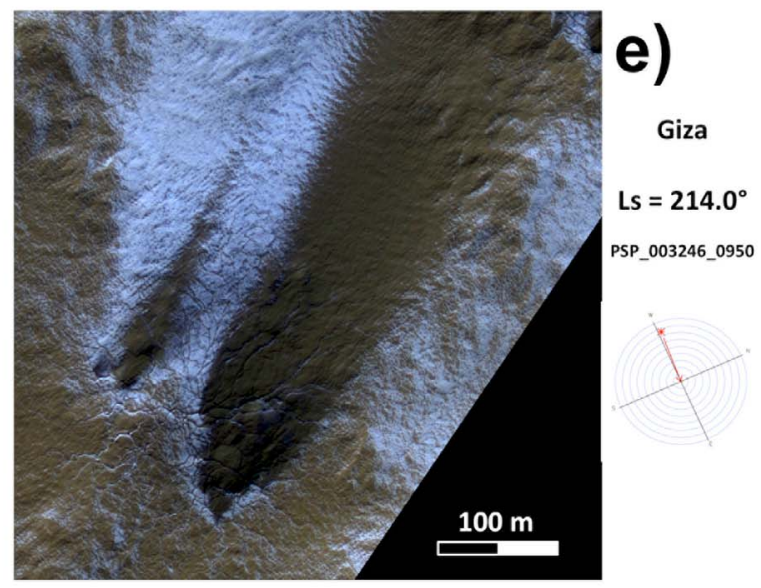

Figure 9. Examples of the "blue haloes" observed by HiRISE between Ls $=183^{\circ}$ and Ls $=214^{\circ}$ in different areas of the southern polar regions. The position of the Sun in the sky is reported in the polar diagram on the right side of each image (see explanations in Figure 8 caption).

disappears from the surface before Ls $=210^{\circ}$ (Figure 11d), and dark fans that had previously disappeared below the blue material are visible again at the same places as before.

[35] Figure 12 shows the evolution of another fixed scene in the region of Ithaca during later spring $\left(\mathrm{Ls}=217^{\circ}\right.$ to $\mathrm{Ls}=$ $252^{\circ}$, step B to C on curves from Figure 4). Contrasting with the dynamic evolutions observed between Ls $=180^{\circ}$ and Ls $=210^{\circ}$, terrains do not show evidence for significant temporal changes. In Figures 12a and $12 \mathrm{~b}\left(\mathrm{Ls}=217^{\circ}\right.$ and $\mathrm{Ls}=$ $224^{\circ}$ ), we can still see small haloes around small dark deposits (top right section of Figures 12a and 12b) but with small contrast compared to haloes observed earlier. An interesting observation in this time series is the reversal of albedo contrast between channels and surrounding flat areas in the bright region: in Figures $12 \mathrm{a}$ and $12 \mathrm{~b}\left(\mathrm{Ls}=217^{\circ}\right.$ and Ls $=224^{\circ}$ ), small channels appear clearly brighter. On the 

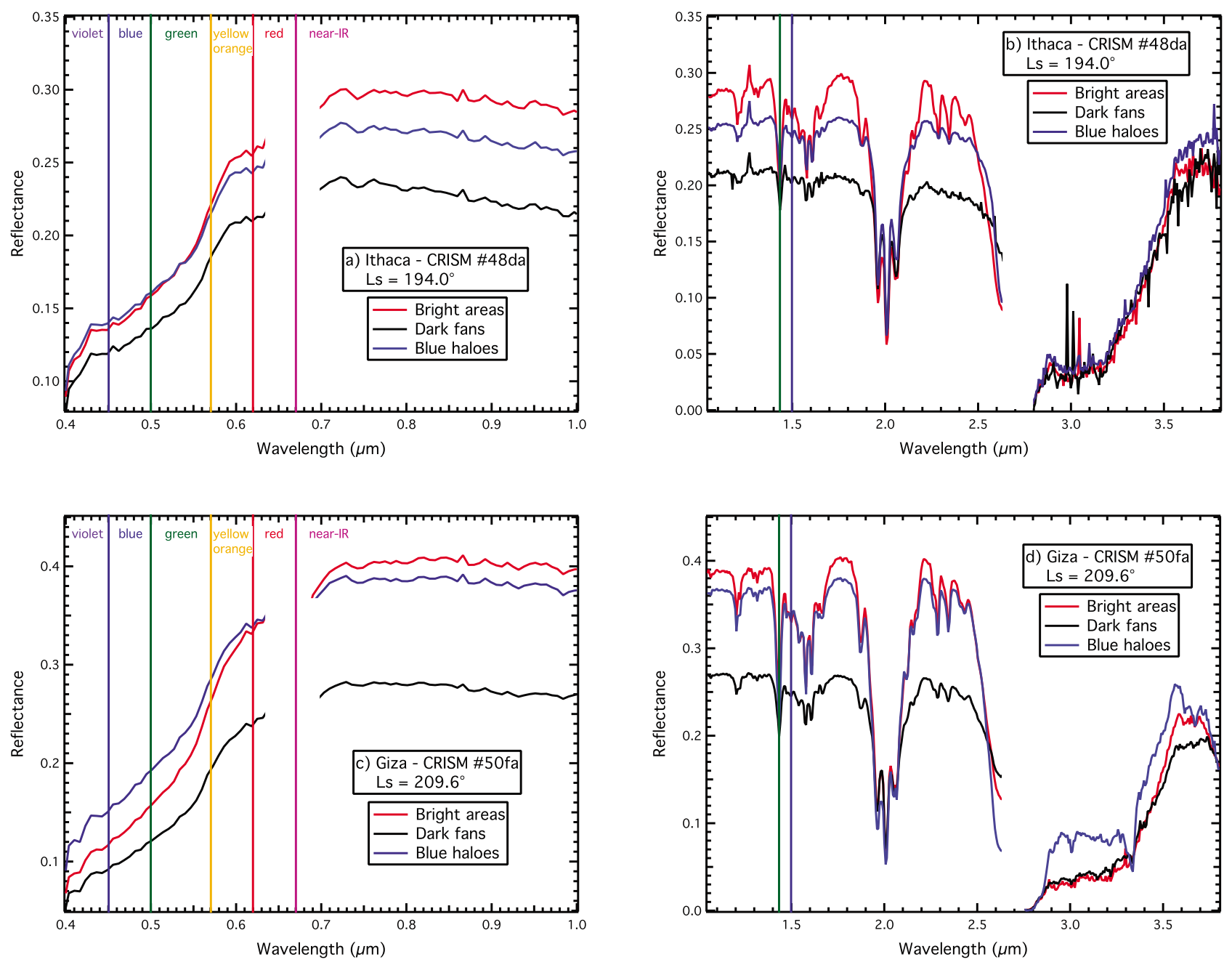

Figure 10. Comparisons of the visible (Figures 10a and 10c) and near-infrared (Figures 10b and 10d) reflectance spectra of the three types of soils observed in the regions of (a and b) Ithaca and (c and d) Giza: bright areas (red), dark fans (black) and blue haloes (blue).

other hand, they appear darker than surrounding areas in Figures $12 \mathrm{~d}$ and $12 \mathrm{e}$ of the series $\left(\mathrm{Ls}=242^{\circ}\right.$ and $\left.\mathrm{Ls}=252^{\circ}\right)$. Figure $12 \mathrm{c}$ acquired at $\mathrm{Ls}=237^{\circ}$ shows an intermediate state with darker channels in the lower right corner and a vast region in the center where channels disappear due to the absence of contrast with surrounding flat ground.

[36] The bright area located between the two big dust fans (top left corner) also shows significant temporal evolution. In Figure $12 \mathrm{a}\left(\mathrm{Ls}=217^{\circ}\right)$, this area shows a bluish color typical of blue haloes previously described. Later, dark channels appear in that area as well as on the left edge of the lower big dust fan. The areas located between the channels brighten in the same time. Finally, at Ls $=252^{\circ}$, most of the areas that were previously affected by this brightening appear dark again, with the same albedo and color as the centers of the dark deposits.

\section{Interpretation and Discussion}

[37] The aim of this section is to interpret and discuss the various high-resolution observations previously presented using the physical principles explained and modeled by
Portyankina et al. [2010a] and Thomas et al. [2010, 2011]. It has been hypothesized by Kieffer et al. [2006] and Kieffer [2007] that the observed jet-like activity is related to the sublimation of the $\mathrm{CO}_{2}$ ice layer "from the bottom" as a result of a self-cleaning process of the icy layer in early spring. This cleaning is proposed to happen by sinking of the embedded dust particles warmed by the Sun through a compact and transparent $\mathrm{CO}_{2}$ ice slab. Increasing pressure at the bottom of the $\mathrm{CO}_{2}$ slab by the basal sublimation eventually results in the breaking of this slab and the emission of a jet of $\mathrm{CO}_{2}$ gas and mineral dust and sand. Portyankina et al. [2010a] physically modeled this self-cleaning to retrieve estimates of the timescales of this process. They show that the time for particles to sink through the ice layer can range between hours and months depending on the particle properties (size, shape, albedo, orientation, etc.). In the same time, the ice layer also sublimes "from the top" as the atmosphere warms up during spring. The temporal evolution of the appearance of the layer of volatile deposits is likely a consequence of a competition between these two processes, with their respective effects changing signifi- 

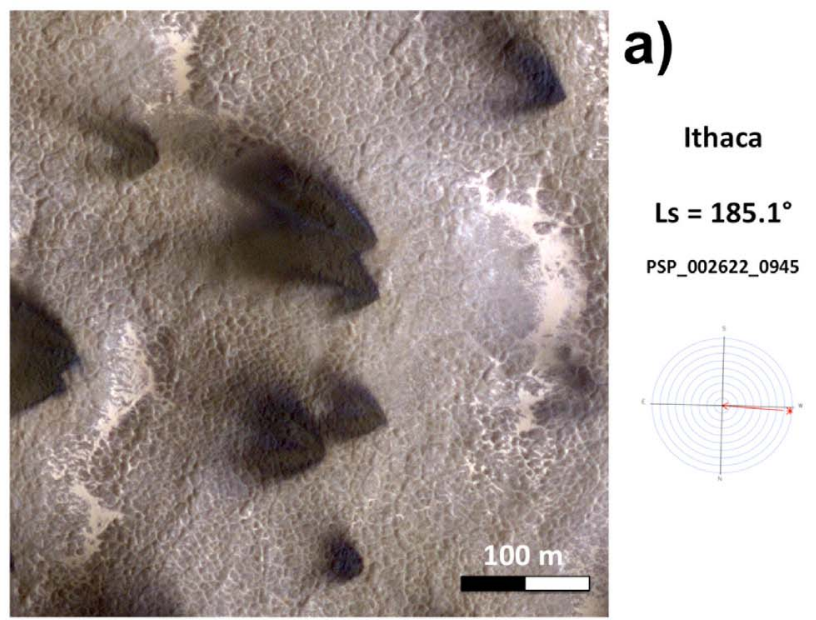

PSP_002622_0945
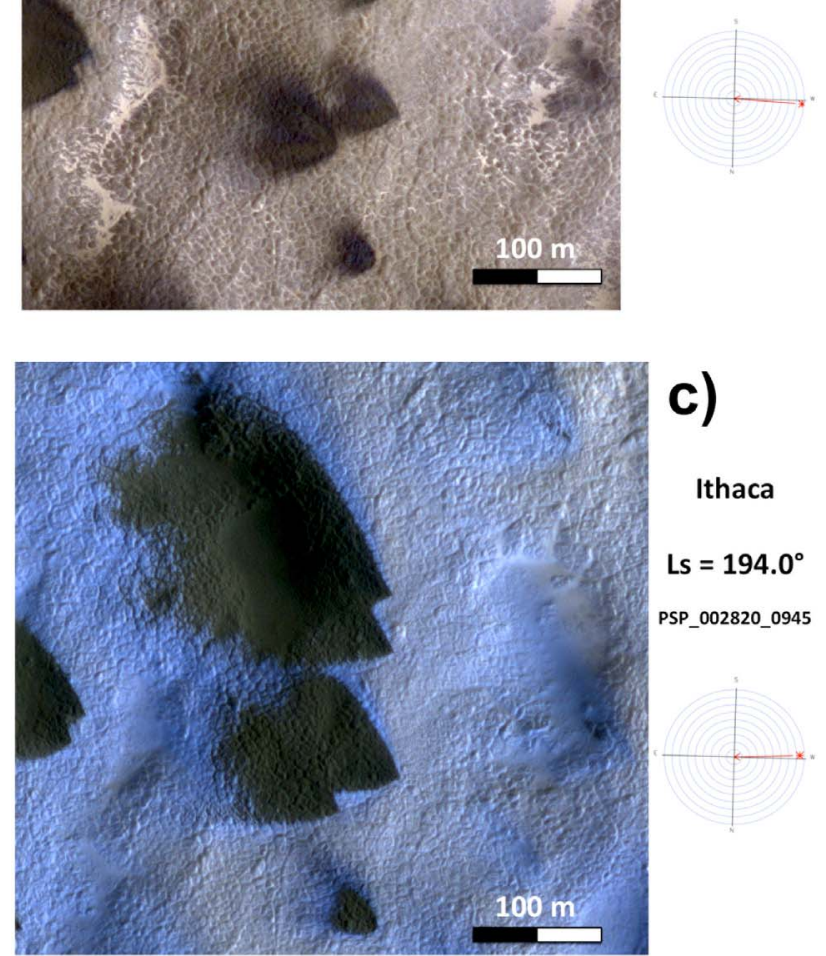

\section{c)}

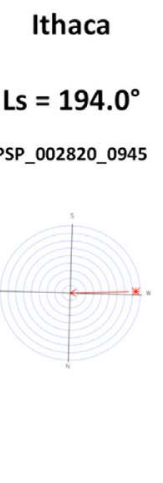

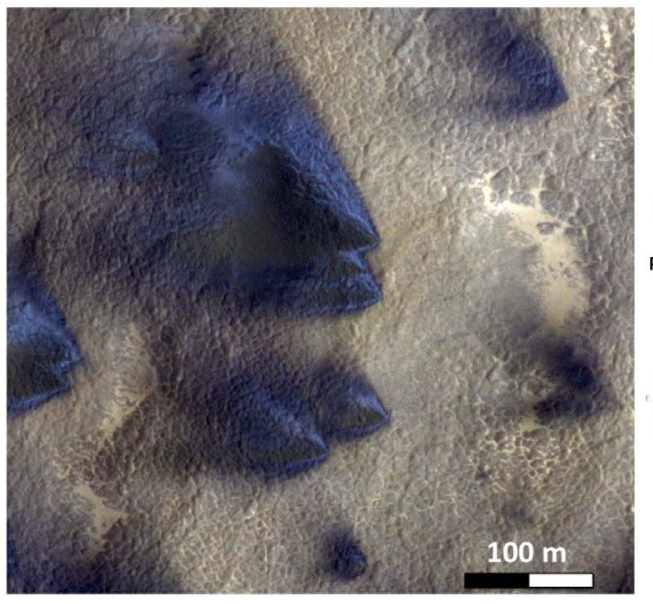

\section{b)}

Ithaca

$L S=187.5^{\circ}$

PSP_002675_0945

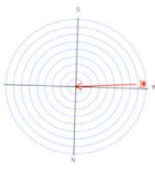

d)

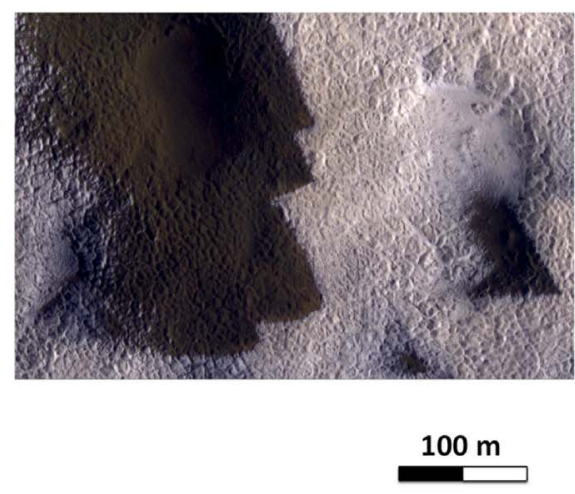

Ithaca

$L S=210.6^{\circ}$

PSP_003176_0945

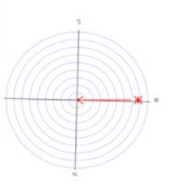

Figure 11. Temporal evolution of a fixed scene in the region of Ithaca between Ls $=185.1^{\circ}$ and $210.6^{\circ}$. Between Ls $=185^{\circ}$ and $194^{\circ}$, the outer edges of the large dark fans become bright and blue. We interpret this evolution as an effect of the sinking of the sand through the $\mathrm{CO}_{2}$ ice layer that leaves behind an optically thin layer of fine-grained $\mathrm{CO}_{2}$ through which the dark grains are still visible (see section 4.2 for interpretation). The position of the Sun in the sky is reported in the polar diagram on the right side of each image (see explanations in Figure 8 caption).

cantly over time. In this section, we successively explore and discuss different steps of this scenario.

\subsection{Early Jet Activity}

[38] Most early HiRISE and CRISM observations (Ls < $190^{\circ}$ ) already show terrain covered by abundant dark deposits, i.e., in most areas, dust fans develop and increase their surface coverage of the icy layer during about 6 to 10 weeks. This increase in covered area of the dust fans is accompanied by a consequent darkening of the fan-free bright areas as a result of the deposition of a fine mobile dust. There exist numerous challenges to conciliate these observations with the classical "Kieffer" model:

[39] 1. The amount of solar energy deposited on the surface in early spring is small because of the high incidence angle. However, the jet activity seems more intense at these early times.

[40] 2. High-resolution targeted CRISM observations do not show the very high value of $\mathrm{CO}_{2}$ band strength (satura- tion of the $1.4 \mu \mathrm{m}$ band [see Langevin et al., 2006, Figure 1]) that would be expected in the case of a perfectly clean $\mathrm{CO}_{2}$ ice slab as hypothesized by Kieffer [2003]. Moreover, we observe a systematic positive correlation between surface albedo and $\mathrm{CO}_{2}$ band strength, both spatially and temporally (Figures 3,6, and 7), in apparent contradiction with the idea of the transparent $\mathrm{CO}_{2}$ slab, as already stated by Titus et al. [2007]. The surface of the icy layer seems highly contaminated by fine mineral dust at the time of CRISM observations, blocking the incident solar radiation and thus limiting the possibility for grains to sink through the entire layer under the effect of sunlight, a concern which was previously raised by Langevin et al. [2006] and Brown et al. [2010], using OMEGA and CRISM "mapping mode" data, respectively.

[41] 3. High spatial heterogeneity at the kilometer scale observed by HiRISE and CRISM is hard to understand in the hypothesis of a global meter thick transparent $\mathrm{CO}_{2}$ slab 

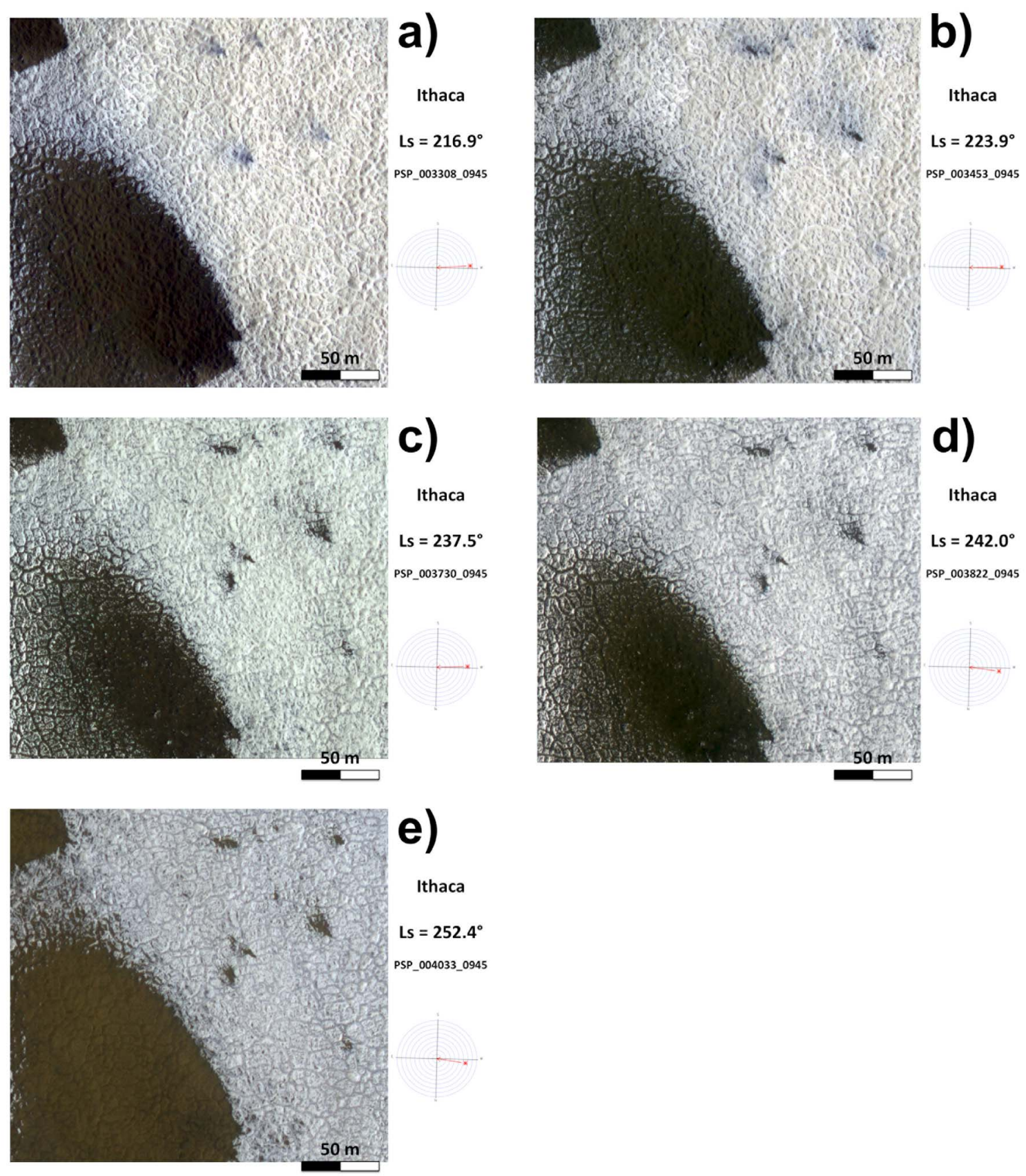

Figure 12. Series of HiRISE pictures in the regions of Ithaca showing the temporal evolution of a dark fan and surroundings bright area between $\mathrm{Ls}=217^{\circ}$ and $252^{\circ}$. The position of the Sun in the sky is reported in the polar diagram on the right side of each image (see explanations in Figure 8 caption).

ice layer, as was previously already discussed using MOC data by Kieffer [2007].

[42] Building upon our observations in an attempt to bypass these apparent contradictions, we propose that the self-cleaning process responsible for the formation of early dust fans occurs very fast at the beginning of spring, only locally, and is controlled by small-scale $(10-100 \mathrm{~m})$ topography (Figure 13). When the very first solar irradiation reaches the surface at the beginning of spring, the $\mathrm{CO}_{2}$ ice is still in the form of a translucent slab formed during winter. Because the Sun is extremely low on the horizon, only a very low amount of solar energy can be deposited into flat ground because of the small projected solar flux (cos $\mathrm{i}$ is small) and the high reflection coefficient of $\mathrm{CO}_{2}$ ice at high incidence angle [Pilorget et al., 2011].

[43] However, any slope oriented toward the Sun will receive a considerably increased amount of peak energy. For an incidence angle of $85^{\circ}$, a $20^{\circ}$ slope oriented toward the Sun receives about 5 times more solar energy than the flat ground. In addition, the reflection coefficient will be about 5 times smaller. As a consequence, with a slope of $20^{\circ}$ only, 25 times more energy can be transmitted through the ice 
compared to a flat ground. The solar energy can then penetrate deep in the slab, and trigger the self-cleaning and jet activity. At the very high latitudes of the studied sites, the solar azimuth varies considerably during the course of the day and slopes with a wide range of orientations can be efficiently illuminated. Moreover, in the case of negative topographic features (like spiders), multiple reflections of light between the walls (of the channels in the case of spiders) could increase the absorption of solar energy in a kind of natural solar oven. In addition to these illumination effects, the presence of slopes can also lead to the existence of mechanical weak points in the $\mathrm{CO}_{2}$ slab ice layer where fracturing and jet activity will preferentially occur. Finally, we don't rule out the possibility that the deposition of $\mathrm{CO}_{2}$ ice in winter is not completely conformal and that spatial variability of the thickness of the $\mathrm{CO}_{2}$ slab over topography might influence its ability to break.

[44] The early jet activity emits a large quantity of mineral material over the surface: dark basaltic sand that settles close to the source of the jet, and red dust that is transported over

\section{a)}

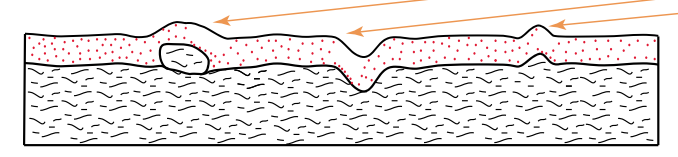

b)
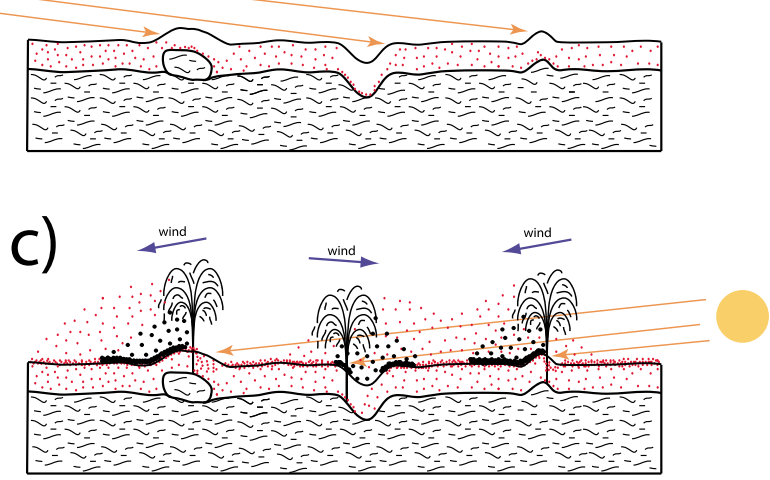

d)

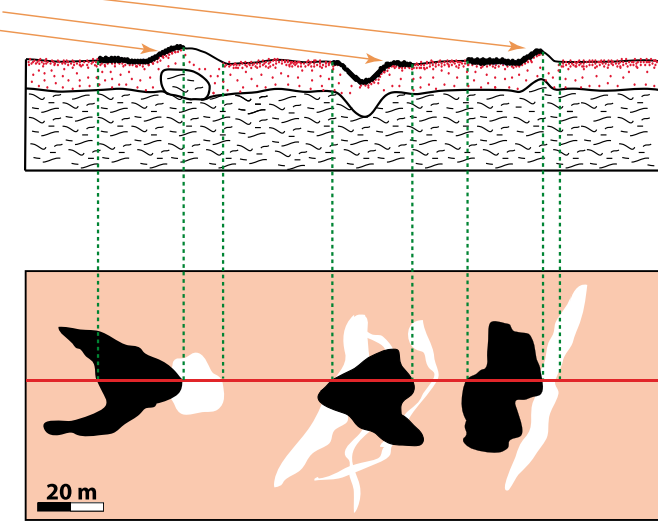

longer distance by local winds and eventually builds a homogeneous thin layer on top of the $\mathrm{CO}_{2}$ slab. The dust and sand that falls back down on the slope can sink through the ice and eventually get back to the ground below the ice. Because the horizontal areas of the $\mathrm{CO}_{2}$ slab layer get rapidly contaminated by dust, the self-cleaning of the ice and subsequent jet activity will always remain less efficient at these locations, even when the Sun will be higher above the horizon later in the season.

[45] In Figures 8 and 11 we show that the presence of dark fans is often spatially associated with the presence of very bright/white areas on slopes. In the framework of the scenario described in this section, these bright slopes are the remnants of the areas affected by self-cleaning. The fact that these areas appear bright in Figures 8 and 11 means that a large part of the solar energy is backscattered and doesn't reach the ground. Earlier, these areas must have appeared as dark as the underlying ground because the $\mathrm{CO}_{2}$ ice was largely transparent. Later, the ice on the slopes becomes brighter because of fracturation, deposition of frost on the surface, or disaggregation of the upper layer in small grains. All processes that might be triggered by the jet activity itself.

[46] The occurrence of jet activity only in areas that display small-scale topography provides an explanation for the strong spatial heterogeneity of the occurrence of the dark deposits on the surface. As already noted by different authors, the most recurrent surface features that show spatial correlation with dark deposits are araneiform features [Hansen et al., 2010]. Thomas et al. [2010] show that the

Figure 13. Four-step scenario for the development of an early springtime jet activity driven by small-scale topography of the ground during step $1(\mathrm{~A} \rightarrow \mathrm{B})$ of the time curves in Figure 4. (a) We represent a homogeneous $\mathrm{CO}_{2}$ ice layer with a conformal topography that is affected by the presence of channels (like in Manhattan), boulders (like in Inca City), and ridges (like in Ithaca). The $\mathrm{CO}_{2}$ ice is in the form of a compact and translucent slab, mostly clean except of some mineral grains (dust size) embedded inside the ice layer. Illumination by the Sun at a very high incidence angle in the first days of spring results in the cleaning of Sun-facing slopes as mineral grains sink through the ice. Close to the geographic pole, the position of the Sun varies by $360^{\circ}$ in the sky during the course of the day, efficiently illuminating slopes with all orientations. (b) We represent the Sun $12 \mathrm{~h}$ later than in Figure 13a, illuminating the opposite slopes of positive and negative morphologic features. The particles of dust embedded into the $\mathrm{CO}_{2}$ ice sink and eventually reach the bottom of the ice layer. (c) As a response to the continuous increase of pressure at the interface between the ice and the ground, the ice layer breaks locally, resulting in jets of $\mathrm{CO}_{2}$ gas and mineral particles as modeled by Thomas et al. [2011]. We represent two populations of grains: large/dark ones ("basaltic sand," in black) that settle close to the vent source and produce dark fans and small/light ones ("iron oxide-rich dust," in red) that are spread over larger areas and contribute to a general decrease of the volatile layer albedo. (d) The resulting appearance of the ice layer in (top) cross section and (bottom) map view displays dark sand fans and bright channels/boulders/ridges slopes. 

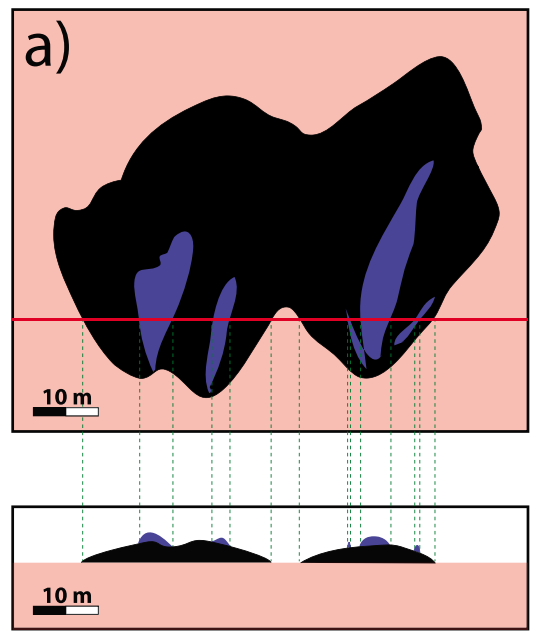
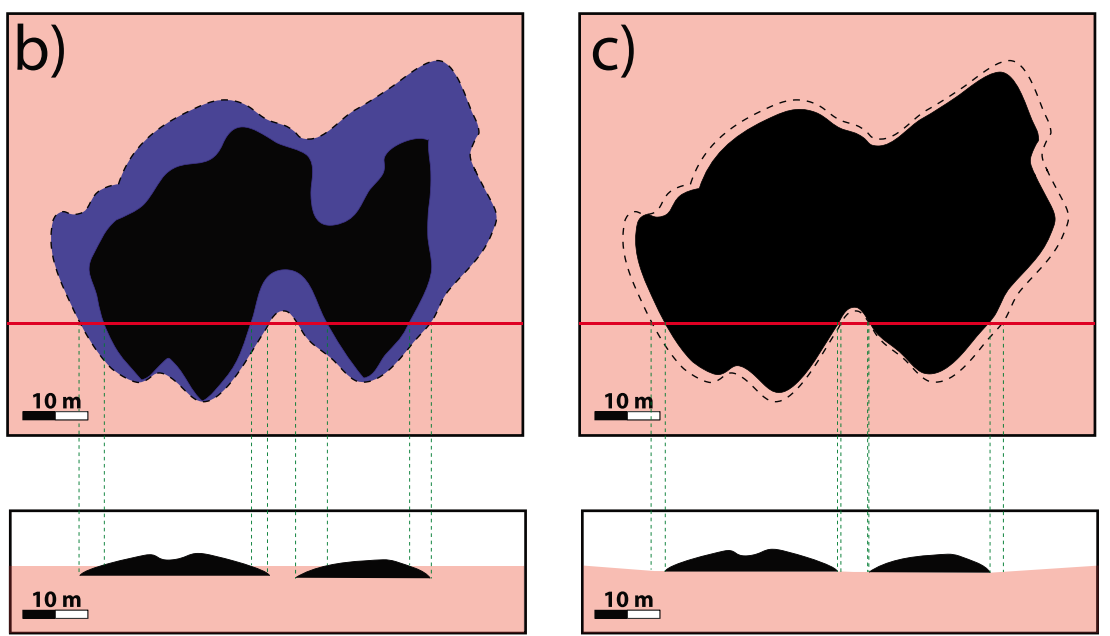

Figure 14. Scenario for the formation of bright blue halos on the outer edge of the dark sand fans around point B of the time curves (Figure 4). (top) Bird's-eye view. (bottom) Cross sections along the horizontal red line drawn on the bird's-eye views. The drawings and spatial scales are based on the shape of actual dust fans observed in the region of Ithaca. (a) In this scenario, we interpret the thin bright/blue stripes in the middle of the fans as thin deposits of $\mathrm{CO}_{2}$ frost resulting from the condensation of $\mathrm{CO}_{2}$ gas emitted by the jet. (b) In contrast, the bluish color of the external edges of the fans is attributed to the sinking of the mineral particles through the ice. (c) Sublimation of the ice layer from the top lets most of the mineral particles reappear, free of $\mathrm{CO}_{2}$ ice with their initial dark albedo, whereas other particles in the most outer part of the fans are still hidden behind an optically thick layer of $\mathrm{CO}_{2}$ ice. This results in a slightly reduced extent of the dark fan compared to its initial state (dashed line).

sources of most dark fans are located close to the radial channels or the edges of the spiders centers rather than the centers themselves as previously stated by Piqueux et al. [2003]. Our observations do not allow us to draw new conclusions about possibility that the araneiform features are actually results of repeated jet activities at the same locations from year to year [Piqueux et al., 2003]. However, these authors also mention the hypothesis that "the channels themselves lead to an increased likelihood of geyser formation, although the reasons for this are unclear." Our hypothesis on the role of channels slopes in the available energies for the jet activity now offers a reasonable explanation for this mechanism.

[47] At larger spatial scale, the south seasonal polar cap shows significant spatial heterogeneity in its temporal evolution with the development of the dark "cryptic region" around Ls $=200^{\circ}-220^{\circ}$ [Kieffer et al., 2000; Langevin et al., 2007]. From our localized high-resolution analyses, it seems likely that the general darkening of the ice is largely due to the emission of fine mineral dust by the jet activity. Contrary to larger grains that settle close to the vent and form dark fan deposits, lighter grains can be transported by the atmosphere over longer distances and settle to form a homogeneous thin mantle that contaminates the top layer of the $\mathrm{CO}_{2}$ seasonal cap. In this respect, the differences in albedo evolution of the cryptic and anticryptic region can easily be connected to the differences in the jet activity intensity inside and outside the cryptic region, with the former being intense and very dense, and the latter more sparse and sporadic.

[48] Beside the early and localized jet activity described in this paragraph, we also observed and studied another type of jet activity characterized by emissions of mineral particles along linear cracks, sometimes forming dark polygonal networks in the ice layer. Similar features were previously observed and analyzed by Kieffer [2007] and Piqueux et al. [2008]. According to the scenario developed in this paragraph, it is the absence of small-scale topography that results in a "polygonal type" activity that is often observed at later time than localized activity in surrounding areas. Observations of the polygonal activity, relations with localized activity and physical processes involved are detailed by G. Portyankina et al. (Polygonal cracks in the seasonal semitranslucent $\mathrm{CO}_{2}$ ice layer in Martian polar areas, submitted to Journal of Geophysical Research, 2011).

\subsection{Formation of the Blue Haloes}

[49] As illustrated in Figures 9, 10 and 11, the formation of blue haloes on the external edges of dark deposits is one of the most spectacular changes affecting the southern polar terrains at springtime. This episode is certainly short although its exact duration is difficult to constrain because it is generally captured on only one or two observations of a given time series, Giza being the most noticeable exception. These haloes were previously observed by Kieffer [2007] on monochromatic MOC images but no clear explanation was proposed for their formation. Piqueux and Christensen [2008] also show an example of a field of so-called "dark spots" observed by HiRISE at Ls $=212^{\circ}$ that actually corresponds to the features we name "blue haloes" in this paper. From studies conducted in the different regions where we see blue haloes, we collected evidence that they usually form on places previously covered by dark deposits, either localized or associated with polygonal fractures, mainly on their external edges. This leads us to the hypothesis that the mineral grains sink through the layer of $\mathrm{CO}_{2}$ ice (Figure 14). The blue color is caused by the mineral grains seen through 
an optically thin layer of $\mathrm{CO}_{2}$. This hypothesis is supported by the analysis of CRISM visible spectra (Figures 10a and 10c) that show the same spectral slopes for the dark fans and blue haloes whereas surrounding icy surfaces display a much steeper visible slope associated with their red color (Figures 9 and 11). The near-infrared part of the spectra also shows that the $\mathrm{CO}_{2}$ spectral signatures inside the blue haloes are intermediate between dark deposits and surrounding bright ground, which is fully consistent with the proposed hypothesis. The strength of the $\mathrm{H}_{2} \mathrm{O}$ ice absorption bands seems to follow the same behavior, indicating that water ice might either sink through the $\mathrm{CO}_{2}$ ice together with dust or sublime during the formation of the blue haloes. It is interesting to note that the variability of the appearance of the blue haloes (for example Ithaca versus Giza see Figures 6 and 7) can be easily explained within the framework of the proposed scenario by variations of the depth at which the dark grains sink through the $\mathrm{CO}_{2}$ ice. If the grains stay at a shallow depth, the haloes will be relatively dark with weak $\mathrm{CO}_{2}$ absorptions (case of Ithaca). If the grains sink deeper, the haloes will be brighter, show stronger $\mathrm{CO}_{2}$ absorptions and last longer (case of Giza).

[50] We also occasionally observe, at earlier times than the blue haloes previously described, blue streaks in the middle or outer parts of the dark fans (Figures $11 \mathrm{a}$ and $11 \mathrm{~b}$ : the two middle size fans in the bottom). We interpret these features as the result of the deposition of fine-grained frost condensed from $\mathrm{CO}_{2}$ gas emitted from the vent source. Thomas et al. [2011] model the process of $\mathrm{CO}_{2}$ gas emission and condensation by jet activity. They propose a mechanism to explain the condensation of $\mathrm{CO}_{2}$ on ground involving local downward air movement triggered by the settling of the mineral dust particles. Whereas the formations of the blue streaks on the dark fans and the blue haloes on the outer edges of the same fans involve very different mechanisms, they result in the same appearance of the surface (the bluish color) due to a similar local stratigraphy: optically thin layer of fine-grained $\mathrm{CO}_{2}$ ice on top of a layer of large and dark mineral grains. Note that in the absence of multiple images of the same scene with sufficient time resolution, it might be very difficult to distinguish between the two mechanisms.

[51] The blue haloes are usually short-lived, with the notable exception of Giza, and most of the parts of the dark fans temporarily hidden behind the $\mathrm{CO}_{2}$ ice reappear after $\mathrm{Ls}=200-210^{\circ}$. However, the entire dust fan doesn't systematically reappear and some external parts can remain hidden until much later time. This is illustrated in the schematics in Figure 14c where we represent a limited decrease in the extent of the dark fan after the blue halo episode. The grains forming the external edges of the fan have sunk behind an optically thick layer of $\mathrm{CO}_{2}$ ice and are either efficiently hidden until the sublimation of the overlying ice layer from the top or may even have sunk down to the bottom of the ice layer. This as well fits to our general hypothesis, as the fan deposit will be thinner at the edges and therefore will be more transparent for solar irradiation, which could render the sinking process to be more efficient.

[52] The facts that blue haloes are short-lived and that dark fans often quickly reappear indicates that sublimation of the volatile layer "from the top" becomes rapidly dominant after the blue halo episode. In other words, the blue haloes episode marks the transition between an earlier time where basal sublimation dominates and a later period where surficial sublimation takes over. Note however, that some of the dark deposits that sunk during the formation of the blue haloes remain hidden behind a thicker layer of $\mathrm{CO}_{2}$ ice for a longer time and only reappear on the surface in late spring. Physical properties of the mineral grains (diameter, shape, porosity...) are likely to influence the depth at which they sink during the formation of the blue haloes and thus the time needed to sublimate the overlying layer of $\mathrm{CO}_{2}$ as explained by Portyankina et al. [2010a].

[53] A recent study of CRISM data of Richardson crater, one of our regions of interest, by Kereszturi et al. [2011] points to a different distribution of ices inside and around the spots and haloes. Contrary to what we observe in all our other studied places, Kereszturi et al. [2011] detect a significant enrichment in $\mathrm{H}_{2} \mathrm{O}$ ice of Richardson crater's haloes. Our own investigations confirm this enrichment. According to the results of the investigations on all other places, the results obtained in Richardson crater seem to be an exception. Richardson crater is located at lower latitude than other areas and is remarkable for the high concentration in water ice at the beginning of Spring. Further studies will be necessary to fully understand the cause of the particular behavior of $\mathrm{H}_{2} \mathrm{O}$ ice at this location.

\subsection{Brightening of the Volatile Layer}

[54] The second phase of the temporal evolution (step B $\rightarrow \mathrm{C}$ in Figures 3 and 4) is characterized by a significant increase of the albedo of all studied terrain as well as a strong increase of the $\mathrm{CO}_{2}$ band strength. These two effects were already observed by Langevin et al. [2007] through the analysis of the OMEGA data set and by Brown et al. [2010] through the analysis of the CRISM "mapping mode" data set. As for the initial darkening of the polar terrain (step $\mathrm{A} \rightarrow \mathrm{B}$ in Figures 3 and 4), a temporal evolution of the $\mathrm{CO}_{2}$ ice particle size cannot explain the observed evolution. If the observed brightening were an effect of a decrease of grain size the strength of the $\mathrm{CO}_{2}$ ice spectral signatures would decrease. Therefore, we interpret this evolution as evidence of cleaning of the surface of the volatile layer. The facts that, in this phase, the sublimation of $\mathrm{CO}_{2}$ is generally not accompanied by jet activity and that the appearance of the surface does not show any drastic change (Figure 12) suggest a dominant surficial sublimation of the $\mathrm{CO}_{2}$ ice of the volatile layer (Figure 15). This is also fully consistent with the fact that the strong contamination of the surface of the volatiles layer by dust doesn't allow the solar radiation to penetrate deep in the ice, preventing the self-cleaning and basal sublimation that triggered the jet activity in the previous phase. In such a case, the dust from inside the ice released by the sublimation of the top layer of the volatile deposits could be first mobilized by surface winds and then carried away over long distances, resulting in the observed cleaning of the surface. The movement of dust over the surface of this ice might be favored by the constant emission of gaseous $\mathrm{CO}_{2}$ that can lift the small mineral dust particles. Large-scale movements of dust could contribute to the strong positive correlation observed between surface albedo and atmospheric dust opacity (Figure 2). The increase of atmospheric opacity during southern spring is indeed restricted to the area covered by seasonal volatiles and is not a general behavior in the southern hemisphere, pointing to 
a)

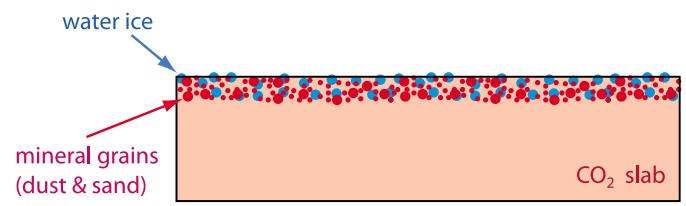

b)

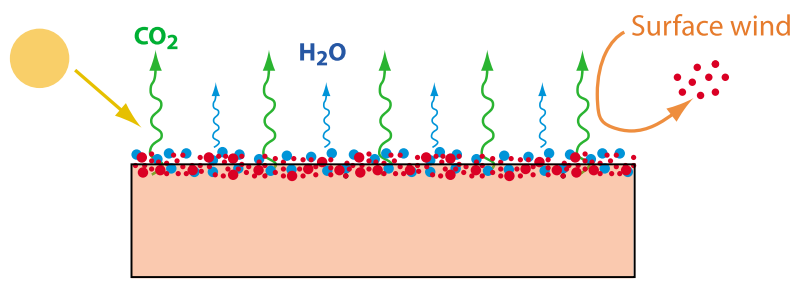

c)

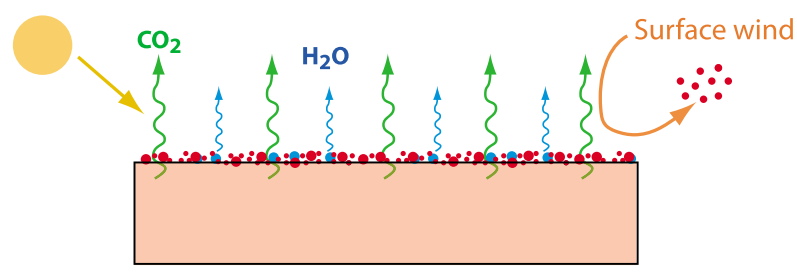

d)

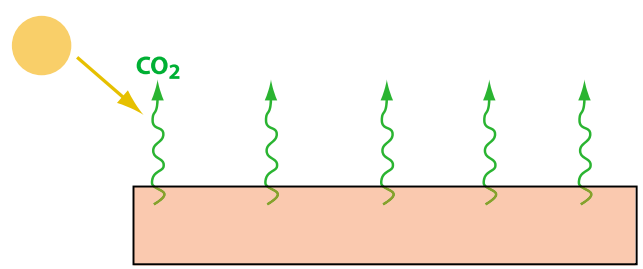

Figure 15. Scenario for the temporal evolutions of surface albedo, $\mathrm{CO}_{2}$ and $\mathrm{H}_{2} \mathrm{O}$ band strength (from CRISM), atmospheric dust content (from OMEGA) and atmospheric $\mathrm{H}_{2} \mathrm{O}$ vapor content (from CRISM) during step $2(\mathrm{~B} \rightarrow \mathrm{C}$ ) of the time curves in Figure 4. During that step, sublimation from the top of the ice layer dominates and releases the embedded mineral dust and water ice particles. These dust particles can be mobilized by surface winds and transported over short to long distances depending on their size and density. As particles are moved away or accumulate in topographic lows, the overall albedo of the ice layer increases considerably. We also represent some of the biggest particles sinking through the ice layer. The continuous flux of $\mathrm{CO}_{2}$ generated by the sublimation of the dry ice leads to the slow sublimation of the particles of $\mathrm{H}_{2} \mathrm{O}$ ice released from the sublimating $\mathrm{CO}_{2}$ in a very low relative humidity atmosphere. We represent a surface layer completely free from $\mathrm{H}_{2} \mathrm{O}$ ice in Figure 15d. This should be understood in a spectroscopic way: there might still be some water ice but in a very low concentration, lower than the detection threshold of the remote sensing technique. the existence of a local source such as the one suggested in this paper. However, an alternative possibility is that the amount of aerosols in the atmosphere over the pole increases as a result of the progressive retreat of the seasonal cap that leaves behind bare soil and dust susceptible to be lifted and transported by winds. It should be possible to distinguish between these hypotheses in the future by directly retrieving local values of atmospheric opacity from the targeted CRISM observations themselves as recently undertaken by Douté et al. [2010].

[55] The predominance of surficial sublimation of the $\mathrm{CO}_{2}$ ice layer at this time (see schematic scenario in Figure 15) is also consistent with results of physical modeling reported by Portyankina et al. [2010a] and with the aforementioned scenario, where the end of the "blue haloes episode" marks the transition between predominance of either basal or surficial sublimation, respectively.

[56] It is also during this phase that the spectral signatures of water ice decrease in strength and finally disappear (Figures 3 and 4). As already stated in section 3, the disappearance of the $\mathrm{H}_{2} \mathrm{O}$ ice signature doesn't necessarily imply that water ice has completely disappeared but that its concentration is below the detection threshold. Thus, water ice might disappear before $\mathrm{CO}_{2}$ ice or together with $\mathrm{CO}_{2}$ ice. The interpretation of this observation is not straightforward because it is in apparent contradiction with the relative sublimation rates of $\mathrm{CO}_{2}$ and $\mathrm{H}_{2} \mathrm{O}$. Chittenden et al. [2008] studied the sublimation rate of $\mathrm{H}_{2} \mathrm{O}$ ice on Mars as a function of temperature and wind speed. They propose an extrapolated relationship between these parameters that, despite large uncertainties, can be used for orders of magnitude calculations. At the temperature of $\mathrm{CO}_{2}$ sublimation on Mars, $145 \mathrm{~K}$, the complete sublimation of a $1 \mu \mathrm{m}$ thick layer of $\mathrm{H}_{2} \mathrm{O}$ ice would last about 100 Martian years!

[57] Knowing the origin of the surface water ice and its physical state inside the $\mathrm{CO}_{2}$ ice layer is also helpful for the interpretation of its temporal evolution. Langevin et al. [2007] propose that the water ice is deposited all over the $\mathrm{CO}_{2}$ ice seasonal deposits around south spring equinox $\left(\mathrm{Ls}=180^{\circ}\right)$ after it has sublimed from a major surface reservoir in Hellas basin. In such a case, $\mathrm{H}_{2} \mathrm{O}$ ice will only contaminate the surface of the $\mathrm{CO}_{2}$ ice layer (together with the significant contamination by mineral dust).

[58] As the sublimation rate of $\mathrm{H}_{2} \mathrm{O}$ ice shows an exponential dependence to the temperature [Chittenden et al., 2008], a moderate warming of $\mathrm{H}_{2} \mathrm{O}$ ice particles by about 20 or $30 \mathrm{~K}$ would be enough to accelerate their sublimation by several orders of magnitude, which would make it consistent with the duration of phase 2. Our preferred hypothesis is that the surficial sublimation of the $\mathrm{CO}_{2}$ ice releases the $\mathrm{H}_{2} \mathrm{O}$ ice and mineral dust particles previously embedded in its top layer. These particles can be thermally isolated from the underlying $\mathrm{CO}_{2}$ ice by the continuous flux of gaseous $\mathrm{CO}_{2}$ produced by the sublimation. $\mathrm{H}_{2} \mathrm{O}$ ice particles get warmer by a few tens of kelvins and slowly sublime, explaining why water ice spectral signatures decreases slowly and continuously, over more than $50^{\circ}$ of Ls in some cases (Giza, Ithaca, Figure 3). This hypothesis is consistent with surface temperature retrievals from THEMIS observations that show that the surface temperature can be warmer than the $\mathrm{CO}_{2}$ sublimation temperature by a few kelvins [Kieffer et al., 2006] consistent with the 
idea that a minor fraction of the surface material is warmer than the $\mathrm{CO}_{2}$ ice.

[59] This evolution strongly contrasts with the one observed in north polar regions where the sublimation of the $\mathrm{CO}_{2}$ ice leaves a layer of $\mathrm{H}_{2} \mathrm{O}$ ice particles at the surface that becomes eventually thick enough to completely hide the spectral signature of $\mathrm{CO}_{2}$ ice [Appéré et al., 2011]. Two parameters can explain this different behavior: the difference of amount of water ice in the volatile layer in the Northern and in the Southern seasonal cap and the difference of relative humidity of the atmosphere during spring, both being probably linked. In the northern hemisphere, the amount of $\mathrm{H}_{2} \mathrm{O}$ ice is relatively large. When the seasonal cap retreats from the subpolar latitudes toward the pole, the sublimation of $\mathrm{CO}_{2}$ leaves behind a millimeter thick annulus of pure water ice that sublimates at a later time, providing water vapor to the atmosphere. This vapor can then recondense as a frost on top of the $\mathrm{CO}_{2}$ layer that is still present at higher latitudes and contribute to an overlying water ice layer together with the larger grains that were previously embedded in the $\mathrm{CO}_{2}$ ice. In the southern hemisphere, in the absence of such a strong source of $\mathrm{H}_{2} \mathrm{O}$ water vapor, the relative humidity remains low enough so that the rare particles of water ice released by the sublimation of the $\mathrm{CO}_{2}$ just sublime and do not build an optically thick layer on top of the $\mathrm{CO}_{2}$ ice.

[60] We should also mention at this point that there is an apparent disagreement between our results and the ones of Langevin et al. [2007] about the time at which the spectral signatures of water ice entirely disappear from the surface. We systematically observe water ice until a later time as provided on OMEGA maps in almost all the studied areas. The OMEGA data used by Langevin et al. [2007] and the CRISM data set used in this study have been obtained in two different Martian years, MY 27 and MY 28, respectively. Thus, an interannual variability of the water ice deposits could explain these differences. Furthermore, the general characteristics of atmospheric dust for these two years have been very different and it is well known that the dust and $\mathrm{H}_{2} \mathrm{O}$ cycles are strongly coupled [Böttger et al., 2004]. Brown et al. [2010] used CRISM "mapping mode" data collected in the same year as the CRISM targeted observation that we analyze here and they found results in agreement with those of Langevin et al. [2007]. However, they used a threshold of 0.125 to display their data of $\mathrm{H}_{2} \mathrm{O}$ band depth. This value was chosen because it removes "random noise" from their $\mathrm{H}_{2} \mathrm{O}$ detection maps. Applying the same threshold to our $\mathrm{H}_{2} \mathrm{O}$ band strength time curves (Figure 3) would result in an earlier disappearance of $\mathrm{H}_{2} \mathrm{O}$ ice in a lot of regions (more consistent with the results of Langevin et al. [2007] and Brown et al. [2010]) or even in a complete absence of $\mathrm{H}_{2} \mathrm{O}$ ice detection over the entire considered time range in some areas. Nevertheless, we think that such a high threshold is not justified in our case. From the continuity and the smoothness of the time curves, we conclude that the signal-to-noise ratio (SNR) is largely sufficient to be confident in the detection of $\mathrm{H}_{2} \mathrm{O}$ ice with a value of the band strength as low as a few percents. This high SNR is likely the result of averaging tens of individual CRISM spectra at each time step (see section 2 and Figure 3).

[61] The difference of the spatial resolution between OMEGA data and CRISM mapping mode data on one hand and CRISM targeted observations on the other hand can also explain at least part of the aforementioned differences. We show from our analyses that the spectral signature of $\mathrm{H}_{2} \mathrm{O}$ ice is much stronger over bright areas than over dark areas. Averaging of the spectra of these two types of surfaces observed at low spatial resolution ("geographic mixture") inevitably results in a decrease of the measured values of $\mathrm{H}_{2} \mathrm{O}$ band strength, eventually preventing the detection of water ice during late spring.

\subsection{Progressive Defrosting}

[62] The last phase of the spring/summer evolution of south polar terrains is the progressive defrosting, usually after Ls $=250^{\circ}$ at latitudes higher than $80^{\circ} \mathrm{S}$. This phase is poorly documented in our study as the regional dust storm in MY 28 prevented good observations of the surface of the south polar terrain between $\mathrm{Ls}=260^{\circ}$ and $\mathrm{Ls}=290^{\circ}$. However, this step has already been the subject of extensive work in the past and is now well understood [Schmidt et al., 2009, 2010].

\section{Conclusion and Perspectives}

[63] HiRISE and CRISM high-resolution observations provide new insights into the processes responsible for the evolution of the appearance of south polar terrains in spring. They complement the results obtained from TES [Kieffer et al., 2000], THEMIS [Kieffer et al., 2006; Kieffer, 2007], OMEGA [Langevin et al., 2007; Schmidt et al., 2009, 2010] and CRISM mapping mode [Brown et al., 2010] data sets, which provide lower spatial resolution but a global coverage of the seasonal deposits.

[64] By deriving time curves of surface reflectance from HiRISE and CRISM data and of $\mathrm{CO}_{2}$ band strength from CRISM data for ten fixed areas at high latitudes, we identified three distinct phases in the evolution of the appearance of polar terrains. The first phase with times before Ls $=200^{\circ}$ consists in a general darkening of the surface of the ice layer, associated with the early apparition and development of dark fans. The second phase, from Ls $=200^{\circ}$ to $260^{\circ}$, corresponds to a brightening of the icy layer associated with a strong increase of the strength of the $\mathrm{CO}_{2}$ ice spectral criterion. The third and last step, later than $\mathrm{Ls}=260^{\circ}$, shows a decrease of both surface reflectance and $\mathrm{CO}_{2}$ band strength until these spectral criteria reach values representative of unfrozen soil: reflectance at $1.14 \mu \mathrm{m}$ between 0.2 and 0.3 and absence of spectral signature of $\mathrm{CO}_{2}$ ice.

[65] Building from the HiRISE and CRISM time curves as well as CRISM time-resolved mapping of surface reflectance and $\mathrm{CO}_{2}$ band strength and analysis of color HiRISE images, we propose plausible scenarios for each step of the evolution based on or adapted from physical processes previously mentioned and described by Kieffer et al. [2000], Kieffer [2007], Piqueux et al. [2003], Piqueux and Christensen [2008], Portyankina et al. [2010a] and Thomas et al. [2010, 2011]. We identify the meter-scale topography of the ground as the main factor controlling the occurrence of early jet activity because of the ability of inclined surfaces to collect much more energy than flat surfaces, allowing for the initiation of an ice self-cleaning process that gives rise to the jet activity in the framework of the 
classical Kieffer model. Often, this small-scale topography consists of radial channels forming surfaces features know as "spiders" [Piqueux et al., 2003] or "araneiform features" [Hansen et al., 2010]. It has previously been proposed that these structures are formed by the progressive erosion of the upper layers of the ground by $\mathrm{CO}_{2}$ gas during the venting process over periods of around $10^{4}$ years where the source of the jets remain at the same position [Piqueux et al., 2008]. The control of the occurrence of the jet activity by channels slopes, as proposed in this article, provides a convenient explanation for this process.

[66] From large-scale observations of the south polar terrains in spring the development of the cryptic region is the most obvious and puzzling evolution [Kieffer et al., 2000; Langevin et al., 2007]. However, our new results from high-resolution observations do not show drastic differences between the temporal behaviors of regions located inside or outside the classical boundaries of the cryptic region. They all display the three-step relative evolution extensively discussed above, albeit absolute values of surface reflectance and $\mathrm{CO}_{2}$ band strength being significantly lower in the cryptic region than in the anticryptic region. It is plausible that the cryptic behavior only differs from the anticryptic behavior by the intensity and/or density of the jet activity. According to our observations, the darkening of the cryptic region around $\mathrm{Ls}=200^{\circ}-210^{\circ}$ is related to the emission of a thin layer of mineral dust and sand to the surface by the jet activity rather than to the development of transparent $\mathrm{CO}_{2}$ slab ice. The transparent $\mathrm{CO}_{2}$ slab is still present at that time but is already hidden by the surficial dust layer. The $\mathrm{CO}_{2}$ ice does only appear clean at the surface very early in Spring, before the rapid onset of jet activity results in its contamination by mineral dust. This interpretation is consistent with the conclusions of Langevin et al. [2006, 2007] and Brown et al. [2010].

[67] Piqueux et al. [2003] mapped the occurrence of spiders and came to the conclusion that they are only observed in the cryptic region. Subsequent observations by HiRISE revealed that spiders are also observed outside this cryptic region. However, the density of these features remains higher in the cryptic region, as well as the intensity of the jet activity. The intricate positive feedbacks between the existence of meter-scale topography (e.g., araneiform features) and the jet activity could provide a possible explanation for the difference between cryptic and anticryptic regions. According to this idea, the rheological and textural properties of the ground play a prominent role in the development of jet activity and associated surface features and could control the occurrence of these processes. More investigations on summer observations of the south polar regions will be necessary to confirm such differences between the properties of the soil in the cryptic and anticryptic regions.

[68] Around Ls $=190^{\circ}-220^{\circ}$, corresponding to the transition between phase 1 and 2 on the surface reflectance time curves, we observe the formation of haloes around dark dust/sand fans with a characteristic blue color on HiRISE color composites. Two processes are likely to explain the formation of these "blue haloes": the condensation of $\mathrm{CO}_{2}$ frost from the gas emitted by the jets as modeled by Thomas et al. [2011] and the cleaning of the top of the ice layer as large dark particles sink through it when heated by the Sun. In some areas where we have successive observations of the same scene with sufficient time resolution, we can show that the second process is dominant. We also highlight the short duration of this episode, as the haloes quickly disappear and let the dark mineral material reappear at the same place as before. Our preferred interpretation of this sequence of events is that a transition occurs at that time. Before this transition, mineral grains can sink through the ice layer. After the transition, fast sublimation of the ice from the top of the layer prevents further possibility for the grains to sink into the ice. The reason for this transition is likely to be the warming up of the lower layer of the atmosphere.

[69] Observations of the surface during phase 2 of the time curves are also consistent with this interpretation: jet activity is usually absent or very sporadic, indicating that the self-cleaning process thought to be responsible for the jet activity during step 1 is no more efficient. Furthermore, we see evidence that the dust previously embedded in the top layer of the ice becomes mobile on the surface and accumulates into topographic lows. Thus, we also make the hypothesis that the positive correlation observed between the surface reflectance measured by HiRISE and the atmospheric opacity measured by OMEGA prior to the global dust storm could be caused by the levitation and mobilization of the finest particle size fractions of mineral dust. Both accumulation of dust in topographic lows and transport over longer distance by surface wind result in the brightening of the surface of the ice layer and the strong increase of $\mathrm{CO}_{2}$ ice band strength with maxima generally observed around $\mathrm{Ls}=250-260^{\circ}$. In the same time, water ice continuously sublimes, which might explain the increase of atmospheric water vapor concentration observed by CRISM around $\mathrm{Ls}=250^{\circ}$.

[70] The last step of the evolution is poorly documented here as the MY 28 dust storm prevented any good observation of the surface between Ls $=260^{\circ}$ and $\mathrm{Ls}=280^{\circ}$. Future analysis of the observations by HiRISE and CRISM during subsequent years will hopefully permit to fill this gap in observations and refine scenarios for this step.

[71] Acknowledgments. Part of this work was funded by the Swiss National Science Foundation. We are grateful to the CRISM Team for releasing the powerful CAT software to handle and analyze CRISM data. We acknowledge two anonymous referees for comments and suggestions that led to a significant improvement of the manuscript.

\section{References}

Appéré, T., B. Schmitt, Y. Langevin, S. Douté, A. Pommerol, F. Forget, A. Spiga, B. Gondet, and J.-P. Bibring (2011), Winter and spring evolution of northern seasonal deposits on Mars from OMEGA/Mars Express, J. Geophys. Res., 116, E05001, doi:10.1029/2010JE003762.

Bibring, J.-P., et al. (2004), Perennial water ice identified in the south polar cap of Mars, Nature, 428, 627-630, doi:10.1038/nature02461.

Böttger, H. M., S. R. Lewis, P. L. Read, and F. Forget (2004), The effect of a global dust storm on simulations of the Martian water cycle, Geophys. Res. Lett., 31, L22702, doi:10.1029/2004GL021137.

Brown, A. J., W. M. Calvin, P. C. McGuire, and S. L. Murchie (2010), Compact Reconnaissance Imaging Spectrometer for Mars (CRISM) south polar mapping: First Mars year of observations, J. Geophys. Res., 115, E00D13, doi:10.1029/2009JE003333.

Chittenden, J. D., V. Chevrier, L. A. Roe, K. Bryson, R. Pilgrim, and D. W. G. Sears (2008), Experimental study of the effect of wind on 
the stability of water ice on Mars, Icarus, 196, 477-487, doi:10.1016/j. icarus.2008.01.016.

Delamere, W. A., et al. (2010), Color imaging of Mars by the High Resolution Imaging Science Experiment (HiRISE), Icarus, 205, 38-52, doi:10.1016/j.icarus.2009.03.012.

Douté, S., and B. Schmitt (1998), A multilayer bidirectional reflectance model for the analysis of planetary surface hyperspectral images at visible and near-infrared wavelengths, J. Geophys. Res., 103, 31,367-31,389, doi:10.1029/98JE01894.

Douté, S., B. Schmitt, Y. Langevin, J.-P. Bibring, F. Altieri, G. Bellucci, B. Gondet, F. Poulet, and the MEX OMEGA Team (2007), South pole of Mars: Nature and composition of the icy terrains from Mars Express OMEGA observations, Planet. Space Science, 55, 113-133, doi:10.1016/j.pss.2006.05.035.

Douté, S., M. Vincendon, Y. Langevin, A. Spiga, and J.-P. Bibring, and the OMEGA Team (2010), Monitoring atmospheric dust opacity at high latitudes on Mars by imaging spectroscopy, Abstract P53E-1572 presented at 2010 Fall Meeting, AGU, San Francisco, Calif., 13-17 Dec.

Feldman, W. C., et al. (2004), Global distribution of near-surface hydrogen on Mars, J. Geophys. Res., 109, E09006, doi:10.1029/2003JE002160.

Fischbacher, G. E., L. J. Martin, and W. A. Baum (1969), Martian polar cap boundaries, final report, part a, contract 951547 to Jet Propulsion Laboratory, Planet. Res. Cent., Lowell Obs., Flagstaff, Ariz.

Giuranna, M., V. Formisano, D. Grassi, and A. Maturilli (2007), Tracking the edge of the south seasonal polar cap of Mars, Planet. Space Sci., 55 , 1319-1327, doi:10.1016/j.pss.2006.12.005.

Hansen, C. J., N. Thomas, G. Portyankina, A. McEwen, T. Becker, S. Byrne, K. Herkenhoff, H. H. Kieffer, and M. Mellon (2010), HiRISE observations of gas sublimation-driven activity in Mars' southern polar regions: I. Erosion of the surface, Icarus, 205, 283-295, doi:10.1016/j.icarus.2009.07.021.

Herkenhoff, K. E., and J. J. Plaut (2000), Surface ages and resurfacing rates of the polar layered deposits on Mars, Icarus, 144, 243-253, doi:10.1006/icar.1999.6287.

James, P. B., G. Briggs, J. Barnes, and A. Spruck (1979), Seasonal recession of Mars' south polar CAP as seen by Viking, J. Geophys. Res., 84, 2889-2922, doi:10.1029/JB084iB06p02889.

James, P. B., H. H. Kieffer, and D. A. Paige (1992), The seasonal cycle of carbon dioxide on Mars, in Mars, edited by H. H. Kieffer et al., pp. 934-968, Univ. of Ariz. Press, Tucson.

Kelly, N. J., W. V. Boynton, K. Kerry, D. Hamara, D. Janes, R. C. Reedy, K. J. Kim, and R. M. Haberle (2006), Seasonal polar carbon dioxide frost on Mars: $\mathrm{CO}_{2}$ mass and columnar thickness distribution, J. Geophys. Res., 111, E03S07, doi:10.1029/2006JE002678.

Kereszturi, A., M. Vincendon, and F. Schmidt (2011), Water ice in the dark dune spots of Richardson crater on Mars, Planet. Space Sci., 59, 26-42, doi:10.1016/j.pss.2010.10.015.

Kieffer, H. H. (2000), Annual punctuated $\mathrm{CO}_{2}$ slab-ice and jets on Mars, paper presented at 2nd International Conference on Mars Polar Science and Exploration, Univ. of Iceland, Reykjavik.

Kieffer, H. H. (2003), Behavior of solid $\mathrm{CO}_{2}$ on Mars: A real zoo, paper presented at 6 th International Conference on Mars, NASA, Pasadena, Calif., 20-25 July.

Kieffer, H. H. (2007), Cold jets in the Martian polar caps, J. Geophys. Res., 112, E08005, doi:10.1029/2006JE002816.

Kieffer, H. H., T. N. Titus, K. F. Mullins, and P. R. Christensen (2000), Mars south polar spring and summer behavior observed by TES: Seasonal cap evolution controlled by frost grain size, J. Geophys. Res., 105, 9653-9699, doi:10.1029/1999JE001136.

Kieffer, H. H., P. R. Christensen, and T. N. Titus (2006), $\mathrm{CO}_{2}$ jets formed by sublimation beneath translucent slab ice in Mars' seasonal south polar ice cap, Nature, 442, 793-796, doi:10.1038/nature04945.

Langevin, Y., S. Douté, M. Vincendon, F. Poulet, J.-P. Bibring, B. Gondet B. Schmitt, and F. Forget (2006), No signature of clear $\mathrm{CO}_{2}$ ice from the 'cryptic' regions in Mars' south seasonal polar cap, Nature, 442, 790-792, doi:10.1038/nature05012.

Langevin, Y., J.-P. Bibring, F. Montmessin, F. Forget, M. Vincendon, S. Douté, F. Poulet, and B. Gondet (2007), Observations of the south seasonal cap of Mars during recession in 2004-2006 by the OMEGA visible/near-infrared imaging spectrometer on board Mars Express, J. Geophys. Res., 112, E08S12, doi:10.1029/2006JE002841.

Lemmon, M. T., et al. (2004), Atmospheric imaging results from the Mars Exploration Rovers: Spirit and Opportunity (2004), Science, 306 1753-1756, doi:10.1126/science.1104474.

Litvak, M. L., I. G. Mitrofanov, A. S. Kozyrev, A. B. Sanin, V. I. Tretyakov, W. V. Boynton, N. J. Kelly, D. Hamara, and R. S. Saunders (2007), Longterm observations of southern winters on Mars: Estimations of column thickness, mass, and volume density of the seasonal $\mathrm{CO}_{2}$ deposit from HEND/Odyssey data, J. Geophys. Res., 112, E03S13, doi:10.1029/ 2006JE002832.
Malin, M. C., et al. (1998), Early views of the Martian surface from the Mars Orbiter Camera of Mars Global Surveyor, Science, 279, 1681-1685, doi:10.1126/science.279.5357.1681

McEwen, A. S., et al. (2007), Mars Reconnaissance Orbiter's High Resolution Imaging Science Experiment (HiRISE), J. Geophys. Res., 112, E05S02, doi:10.1029/2005JE002605.

Morgan, F., F. Seelos, S. Murchie, and the CRISM Team (2009), CRISM data users' workshop CAT tutorial, paper presented at the MRO/CRISM Data Users' Workshop 2009, Johns Hopkins Univ. Appl. Phys. Lab., The Woodlands, Tex.

Murchie, S., et al. (2007), Compact Reconnaissance Imaging Spectrometer for Mars (CRISM) on Mars Reconnaissance Orbiter (MRO), J. Geophys. Res., 112, E05S03, doi:10.1029/2006JE002682.

Pilorget, C., F. Forget, E. Millour, M. Vincendon, and J.-B. Madeleine (2011), Dark spots and cold jets in the polar regions of Mars: New clues from a thermal model of surface $\mathrm{CO}_{2}$ ice, Icarus, 213, 131-149, doi:10.1016/j.icarus.2011.01.031.

Piqueux, S., and P. Christensen (2008), North and south subice gas flow and venting of the seasonal caps of Mars: A major geomorphological agent, J. Geophys. Res., 113, E06005, doi:10.1029/2007JE003009.

Piqueux, S., S. Byrne, and M. I. Richardson (2003), Sublimation of Mars's southern seasonal $\mathrm{CO}_{2}$ ice cap and the formation of spiders, J. Geophys. Res., 108(E8), 5084, doi:10.1029/2002JE002007.

Piqueux, S., C. S. Edwards, and P. R. Christensen (2008), Distribution of the ices exposed near the south pole of Mars using Thermal Emission Imaging System (THEMIS) temperature measurements, J. Geophys. Res., 113, E08014, doi:10.1029/2007JE003055.

Plaut, J. J., et al. (2007), Subsurface radar sounding of the south polar layered deposits of Mars, Science, 316, 92-95, doi:10.1126/ science. 1139672

Portyankina, G., W. J. Markiewicz, N. Thomas, C. J. Hansen, and M. Milazzo (2010a), HiRISE observations of gas sublimation-driven activity in Mars' southern polar regions: III. Models of processes involving translucent ice, Icarus, 205, 311-320, doi:10.1016/j.icarus.2009.08.029.

Portyankina, G., M. Vincendon, N. Thomas, and K.-M. Aye (2010b), Recovery of surface reflectance from atmospheric aerosol contribution: Application to HiRISE images, Lunar Planet. Sci., 41st, Abstract 1533. Prettyman, T. H., W. C. Feldman, and T. N. Titus (2009), Characterization of Mars' seasonal caps using neutron spectroscopy, J. Geophys. Res., 114, E08005, doi:10.1029/2008JE003275.

Schmidt, F., S. Douté, B. Schmitt, M. Vincendon, J.-P. Bibring, and Y. Langevin, and the OMEGA Team (2009), Albedo control of seasonal South Polar cap recession on Mars, Icarus, 200, 374-394, doi:10.1016/j. icarus.2008.12.014

Schmidt, F., B. Schmitt, S. Douté, F. Forget, J.-J. Jian, P. Martin, Y. Langevin, and J.-P. Bibring, and the OMEGA Team (2010), Sublimation of the Martian $\mathrm{CO}_{2}$ seasonal South polar cap, Planet. Space Sci., 58, 1129-1138, doi:10.1016/j.pss.2010.03.018.

Smith, D. E., M. T. Zuber, and G. A. Neumann (2001), Seasonal variations of snow depth on Mars, Science, 294, 2141-2146, doi:10.1126/ science. 1066556 .

Smith, M. D. (2004), Interannual variability in TES atmospheric observations of Mars during 1999-2003, Icarus, 167, 148-165, doi:10.1016/j. icarus.2003.09.010.

Smith, M. D., M. J. Wolff, R. T. Clancy, and S. L. Murchie (2009), Compact Reconnaissance Imaging Spectrometer observations of water vapor and carbon monoxide, J. Geophys. Res., 114, E00D03, doi:10.1029/ 2008JE003288

Tanaka, K. L., and E. J. Kolb (2001a), Geologic history of the polar regions of Mars based on Mars Global Surveyor data: I. Noachian and Hesperian periods, Icarus, 154, 3-21, doi:10.1006/icar.2001.6675.

Tanaka, K. L., and E. J. Kolb (2001b), Geologic history of the polar regions of Mars based on Mars Global Surveyor data: II. Amazonian period, Icarus, 154, 3-21, doi:10.1006/icar.2001.6675.

Tanaka, K. L., and D. H. Scott (1987), Geologic map of the polar regions of Mars, U.S. Geol. Surv. Misc. Invest. Ser., Map I-1802-C, scale $1: 15,000,000$

Thomas, N., C. J. Hansen, G. Portyankina, and P. S. Russel (2010), HiRISE observations of gas sublimation-driven activity in Mars' southern polar regions: II. Surficial deposits and their origins, Icarus, 205, 296-310, doi:10.1016/j.icarus.2009.05.030.

Thomas, N., G. Portyankina, C. J. Hansen, and A. Pommerol (2011), HiRISE observations of gas sublimation-driven activity in Mars' southern polar regions: IV. Fluid dynamics models of $\mathrm{CO}_{2}$ jets, Icarus, 212, 66-85, doi:10.1016/j.icarus.2010.12.016

Titus, T. N., Y. Langevin, S. L. Murchie, F. Seelos, and H. H. Kieffer, and the CRISM Science Team (2007), MRO CRISM observations of the south polar cryptic region, paper presented at Seventh International Conference on Mars, NASA, Pasadena, Calif. 
Vincendon, M., Y. Langevin, F. Poulet, J.-P. Bibring, and B. Gondet (2007), Recovery of surface reflectance spectra and evaluation of the optical depth of aerosols in the near-IR using a Monte Carlo approach: Application to the OMEGA observations of high-latitude regions of Mars, J. Geophys. Res., 112, E08S13, doi:10.1029/2006JE002845.

Vincendon, M., Y. Langevin, F. Poulet, J.-P. Bibring, B. Gondet, and D. Jouglet, and the OMEGA Team (2008), Dust aerosols above the south polar cap of Mars as seen by OMEGA, Icarus, 196, 488-505, doi:10.1016/j.icarus.2007.11.034.

Vincendon, M., Y. Langevin, S. Douté, and J.-P. Bibring (2009), 3 Mars years of dust optical depth mapping by OMEGA above the south pole, paper presented at Third International Workshop on Mars Polar Energy Balance and the $\mathrm{CO}_{2}$ Cycle, Lunar Planet. Inst., Seattle, Wash.
Zuber, M. T., R. J. Phillips, J. C. Andrews-Hanna, S. W. Asmar, A. S. Konopliv, F. G. Lemoine, J. J. Plaut, D. E. Smith, and S. E. Smrekar (2007), Density of Mars' south polar layered deposits, Science, 317, 1718-1719.

K.-M. Aye, A. Pommerol, G. Portyankina, and N. Thomas, Physikalisches Institut, Universität Bern, Sidlerstrasse, 5, CH-3012 Bern, Switzerland. (antoine.pommerol@space.unibe.ch)

C. J. Hansen, Planetary Science Institute, 700 East Fort Lowell, Ste. 106 Tucson, AZ 85719-2395, USA.

Y. Langevin and M. Vincendon, Institut d'Astrophysique Spatiale, CNRS/Université Paris XI, Centre Universitaire d'Orsay, Bat. 120-121, F-91405 Orsay, France. 